\title{
Magnitude and Breadth of Antibody Cross-reactivity Induced by Recombinant Influenza Hemagglutinin Trimer Vaccine Is Enhanced by Combination Adjuvants
}

Jenny Hernandez-Davies

University of California

Emmanuel Dollinger

University of California

Egest Pone

University of California

Jiin Felgner

University of California

Shirin Strohmeier

Icahn School of Medicine at Mount Sinai

Sharon Jan

University of California

Tyler Albin

University of California

Aarti Jain

University of California

Rie Nakajima

University of California

Algimantas Jasinskas

University of California

Florian Krammer

Icahn School of Medicine at Mount Sinai

Aaron Esser-Kahn

University of Chicago

Philip Felgner

University of California

Qing Nie

University of California

Huw DAVIES ( $\nabla$ ddavies@uci.edu )

University of California 


\section{Research Article}

Keywords: toll-like receptor (TLR), IFNg, H5/IVAX-1, IVAX-1, IgG2c

Posted Date: December 13th, 2021

DOl: https://doi.org/10.21203/rs.3.rs-1104187/v1

License: (c) (i) This work is licensed under a Creative Commons Attribution 4.0 International License. Read Full License 


\section{Abstract}

The effects of adjuvants for increasing the immunogenicity of influenza vaccines are well known. However, the effect of adjuvants on increasing the breadth of cross-reactivity is less well understood. In this study we have performed a systematic screen of different toll-like receptor (TLR) agonists, with and without a squalene-in-water emulsion on the immunogenicity of a recombinant trimerized hemagglutinin $(\mathrm{HA})$ vaccine in mice after single-dose administration. Antibody $(\mathrm{Ab})$ cross-reactivity for other variants within and outside the immunizing subtype (homosubtypic and heterosubtypic cross-reactivity, respectively) was assessed using a protein microarray approach. Of all the formulations tested, a combination of CpG, MPLA and AddaVAX (termed "IVAX-1") yielded the greatest breadth and magnitude of Ab responses, particularly against the HA1 region (which includes the variable head domain) of HA. Antigen-specific plasma cell labeling experiments show the components of IVAX-1 are synergistic. This adjuvant preferentially stimulates CD4 T cells to produce Th1 $>$ Th2 type (IgG2c>lgG1) antibodies and cytokine responses. Moreover, IVAX-1 induces identical homo- and heterosubtypic IgG and IgA crossreactivity profiles when administered intranasally. Consistent with these observations, a single-cell transcriptomics analysis demonstrated significant increases in expression of $\lg G 1$, IgG2b and IgG2c genes of $B$ cells in H5/IVAX-1 immunized mice relative to naïve mice, as well as significant increases in expression of the IFNg gene of both CD4 and CD8 T cells. These data support the use of adjuvants for enhancing the breath and durability of antibody responses of influenza virus vaccines.

\section{Introduction}

Despite seasonal vaccination, influenza virus remains a major source of annual morbidity and mortality, and a constant pandemic threat. Influenza A virus (IAV) evolves as it replicates in human and animal hosts owing to an error-prone RNA polymerase that lacks proof-reading function, randomly generating amino acid substitutions in viral proteins. Under selection by the immune response, mutants emerge that escape existing immunity (1-4). The major target of neutralizing antibodies (nAbs) elicited by infection or vaccination is hemagglutinin $(\mathrm{HA})$ on the virion surface, which functions on the virion as a receptor for cell-surface sialic acid to mediate viral entry into host cells $(5,6)$. Thus far, 16 distinct HA subtypes have been found in IAV isolated from mammalian and avian hosts, and two more from bats $(7,8)$. A secondary target of nAbs is neuraminidase (NA), a viral-surface enzyme that facilitates egress from infected cells. There are 11 known NA subtypes. Immune escape is caused by the accumulation of amino acid substitutions in epitopes recognized by nAbs, a process known as antigenic drift $(3,9,10)$. This process has led to the emergence of many more drifted variants of HA than of NA, and lower levels of antibodies induced against NA.

The segmented RNA genome of influenza virus can also lead to the wholesale exchange of different HA subtypes in cells co-infected with two or more different subtypes of influenza virus (11), a process known as antigenic shift. Currently only influenza $A$ viruses expressing $\mathrm{H} 1$ and $\mathrm{H} 3$ subtypes are circulating in humans. However, exchange with a novel HA subtype to which humans have not been previously exposed has the potential to cause pandemics. For example, the highly pathogenic avian influenza virus H5N1 
A/goose/Guangdong/1996 lineage is endemic in poultry in several countries and has caused sporadic lethal infections in humans (12). The antigenic novelty of these viruses pose a continued risk for pandemic potential in naïve human populations $(13,14)$. Pre-pandemic preparedness involves stockpiling vaccines against potential pandemic IAVs. Ultimately, however, it is unknown which subtype will lead to the next pandemic. Thus, the induction of broad protection is as important for pandemic vaccines as it is for seasonal vaccines.

Protection afforded by seasonal vaccination is short-lived, necessitating revision of the vaccine strains on an annual basis. Effectiveness against circulating strains varies year-to-year, anywhere from $0 \%-60 \%$, depending on the accuracy with which the vaccine strains predicted correspond to the strains that are eventually seen in circulation (15), and the overall immune competence of the vaccinee $(16,17)$. Both of these limitations conspire to reduce vaccine efficacy and durability, which ultimately leads to varying levels of morbidity and mortality in susceptible populations. It is likely effectiveness could be improved by a broadly-protective seasonal vaccine which targets conserved epitopes shared by variants (18-21).

In this study, we administered mice a recombinant trimerized HA (either H5 from A/Vietnam/1203/04 or H7 from A/Anhui/1/2013) with 8 different toll-like receptor (TLR) agonists, with or without an oil-in water emulsion, to assess immunogenicity and breadth of the response. All of the adjuvants tested enhanced these responses over antigen alone to varying levels and with different Th1/Th2 ratios. A combination adjuvant we refer to as "IVAX-1", comprising synthetic oligodeoxynucleotides containing unmethylated cytosine-guanine motifs (CpG ODNs) and monophosphoryl lipid A (MPLA) (TLR9 and 4 agonists, respectively) with emulsion induced among the broadest $A b$ responses by microarray. The response was skewed towards Th1 (IgG2c>lgG1) and included nAbs after boosting. A single-cell transcriptomic analysis of whole spleens from mice receiving HA/IVAX-1 confirmed the polarization seen by immunological methods, with additional insights into the spectrum of effects of IVAX-1 during a virus neutralizing response.

\section{Materials And Methods}

2.1 Antigens. Trimerized HA from A/Vietnam/1203/04 (H5N1) or A/Anhui/1/2013 (H7N9) were expressed in insect cells and purified on nickel chelate columns as described (22). Full-length HA0 and HA1 regions for protein microarray printing, B cell labeling and T cell recall assays, were purchased from Sino Biological Inc. (Beijing, China).

2.2 Adjuvants. Tri-agonist TLR 2/6_4_7 was synthesized by conjugation reactions (amide bond formation, maleimide-thiol Michael addition and azide-alkyne click chemistry) of individual TLR agonists to a central triazine as described previously (23). The individual agonists comprised $\mathrm{Pam}_{2} \mathrm{CSK}_{4}$, a TLR2 and TLR6 agonist (TLR2/6 a), pyrimido-indole (TLR4 a), and 2Bxy, an imidazoquinoline derivative (TLR 7 a). TLR triagonist and individual TLR agonists were purified by either high performance liquid chromatography or gel extraction, and their masses were confirmed by matrix-assisted laser desorption/ionization- time of flight (MALDI-TOF) or electrospray ionization-mass spectrometry. The CpG-1018 (TLR9a) was purchased 
from Integrated DNA Technologies (Coralville, lowa) and dissolved in sterile water at $1 \mathrm{mM}$ as stock. Monophosphoryl lipid A (MPLA, TLR4 a) was purchased from Avanti Polar Lipids Inc., (Alabaster, AL; purity $>99 \%$ ). Since MPLA has limited solubility in aqueous solution, this agonist was integrated into liposomes of the inert colipid 1,2-dioleoyl-sn-glycero-3-phospho-(1'-rac-glycerol) (DOPG; Avanti Polar Lipids Inc., Alabaster, $A L$ ) an at 1:5 molar ratio. A squalene oil-in-water emulsion (AddaVAX ${ }^{\mathrm{TM}}$ ) was purchased from Invivogen Inc. (San Diego, CA). Absence of endotoxin (endotoxin activity of $<1 \mathrm{EU} / \mathrm{mL}$ ) in TLRa samples, with the exception of MPLA, was confirmed with LAL Endotoxin Assay Kits (Genscript, Piscataway, NJ) or by HEK TLR4 reporter cell assay (Invivogen, San Diego, CA). MPLA is manufactured in organic solvents and has minimal endotoxin activity.

2.3 Mouse immunizations. All animal work was approved by the UCl Institutional Animal Care and Use Committee (IACUC protocol No. AUP-18-096) and by the Animal Care and Use Review Office (ACURO) of the U.S. Army Medical Research and Materiel Command (USAMRMC). The laboratory animal resources at $\mathrm{UCl}$ are Internationally accredited by the Association for Assessment and Accreditation of Laboratory Animal Care (AAALAC No. 000238). All experiments were performed in accordance with the animal use protocol approved by IACUC and ACURO. This article also follows recommendations in the ARRIVE guidelines for reporting of animal research (24). Female C57BI/6 mice (7-10 weeks of age) were purchased from Charles River Inc., and housed in standard cages with enrichment. Each vaccine dose comprised 2.5mg trimerized HA antigen with different TLR agonists (Table 1). Formulations were used without or with an equal volume of AddaVAX and administered via the sub-cutaneous (s.c.) route ( $50 \mathrm{~mL}$, base of tail) under brief anesthesia with inhaled isofluorane $/ \mathrm{O}_{2}$ mixture. Mice were weighed and monitored daily for the first two weeks for any changes in behavior or appearance and periodically thereafter. Blood was collected into heparinized microcapillary tubes at regular time points (d0, d10, d28, d43) by facial vein bleed under anesthesia with inhaled isofluorane $/ \mathrm{O}_{2}$ and at the experimental endpoint (d103) by cardiac puncture under terminal anesthesia. Tissues (spleens, lymph nodes) were harvested post-mortem for $\mathrm{T}$ cell recall assays.

2.4 Protein Microarrays. HA protein microarrays were fabricated as previously described (25). Briefly, purified influenza HAs spanning 18 influenza A virus subtypes expressed in human or insect cells as either HAO or HA1 molecules, were purchased from Sino Biological Inc. (Beijing, China). Each lyophilized antigen was reconstituted to a concentration of $0.1 \mathrm{mg} / \mathrm{ml}$ in phosphate-buffered saline (PBS) with $0.001 \%$ Tween 20 (T-PBS) and printed onto nitrocellulose-coated glass AVID slides (Grace Bio-Labs, Inc., Bend, OR, USA) using an Omni Grid 100 microarray printer (Genomic Solutions). For probing, plasma samples were diluted 1:100 in protein array blocking buffer (GVS, Sanford, ME, USA) supplemented with a poly-histidine peptide (HHHHHHHHHHGGGG) (Biomatik USA, Delaware, USA) to final concentration $0.10 \mathrm{mg} / \mathrm{ml}$, and preincubated at room temperature (RT) for $30 \mathrm{~min}$. Concurrently, arrays were rehydrated in blocking buffer for $30 \mathrm{~min}$ prior to replacement of the blocking buffer with pre-incubated plasma. Arrays were incubated overnight at $4^{\circ} \mathrm{C}$ with gentle agitation. After washing 6 times with Tris-buffered saline (TBS) containing $0.05 \%$ Tween 20 (T-TBS) at RT, arrays were incubated for $2 \mathrm{~h}$ at RT with biotinylated anti-mouse IgG (Jackson ImmunoResearch; Cat No.115-068-071) or anti-mouse IgA (Kirkegaard \& Perry Laboratories [KPL]; Cat. No. 16-18-01). Arrays were then washed 6 times with T-TBS, followed by incubation with 
streptavidin-conjugated Qdot-800 (Life technologies; Cat. No. Q10173MP) diluted 1:250 in blocking buffer for $1 \mathrm{~h}$ at RT. For IgG subtyping, anti-mouse IgG1-Alexa Fluor ${ }^{\circledR} 647$ or IgG2c-Alexa Fluor555® (Southern Biotech; Cat. Nos. 1073-31 and 1077-32) were used. Arrays were washed three times each with T-TBS followed by TBS, dipped in distilled water, and air-dried by centrifugation at $500 \times \mathrm{g}$ for $10 \mathrm{~min}$. Images were acquired using the ArrayCAM ${ }^{\circledR}$ imaging system (Grace Bio-Labs Inc., Bend, OR) with gain and exposure times of 50 and $400-1000 \mathrm{~ms}$, respectively. Spot and background intensities were measured using an annotated grid (.gal) file. Signal intensities (SI) for each antigen on the array were first background corrected by subtracting sample-specific T-PBS buffer signals from purified protein spot signals.

2.5 Hemagglutination inhibition (HI) assays. Hl assays were performed essentially as described (26). Mouse plasma samples were tested in duplicate by first treating $10 \mu \mathrm{L}$ plasma with $30 \mu \mathrm{L}$ receptor destroying enzyme (RDE; Denka Seiken, Inc.) for $18 \mathrm{~h}$ at $37^{\circ} \mathrm{C}$, after which $30 \mu \mathrm{L}$ of $2.5 \%$ sodium citrate was added and heated at $56^{\circ} \mathrm{C}$ for 30 minutes (27). The volume was brought up to $100 \mu \mathrm{L}$ with PBS, pH7.2 to give a starting dilution of $1 / 10$. Duplicate serial dilutions of $25 \mu \mathrm{L}$ plasma/well were performed across a Vbottomed microtiter plate (Thermo Fisher) in HI assay buffer (FTA Hemagglutination Buffer, Fisher Scientific) and an equal volume of virus (diluted to 4 hemagglutination units [HAU]/25 $\mu \mathrm{L} \mathrm{HI}$ assay buffer) added for $30 \mathrm{~min}$ at RT to allow neuralization to occur. One row without plasma or virus (HI assay buffer only) and one row of without plasma (virus only) were also included as controls. To each well was added $50 \mathrm{~mL}$ 0.5\% freshly-washed turkey red blood cell suspension (Rockland Immunochemicals, Inc., Limerick, PA) in HA buffer and left for 45 mins at $4^{\circ} \mathrm{C}$ to allow red cell pellets to form. The neutralization titer was defined as the reciprocal of the last dilution in which a clear pellet was seen, and multiplied by 10 to correct for the initial dilution from RDE treatment. An HI titer of $1 / 40$ is considered the cut-off for protection in humans (28). Positive control plasma for neutralization assays were produced against reassortant H5N1 virus (obtained from NIBSC, UK, Cat. No. NIBRG-14) in C57BI/ 6 mice by administering 5 $\times 10^{4} \mathrm{TCID}_{50}$ (defined below) in $10 \mathrm{~mL}$ via i.n. route followed 2 weeks later by the same dose administered in $100 \mathrm{~mL}$ via intraperitoneal (i.p.) route. $\mathrm{TCID}_{50}$ is the tissue-culture infective dose, i.e., dilution of virus that caused a cytopathic effect in $50 \%$ of wells containing Madin-Darby canine kidney (MDCK) cells (27).

2.6 Microneutralization $(M N)$ assays. MN assays were performed as described (27) with modifications. Briefly, MDCK cells were obtained from ATCC (Manassas, VA; Cat. No. CCL-34) and maintained in Eagle's minimum essential medium (EMEM; ATCC Cat. No. 30-2003) containing penicillin/streptomycin (Thermofisher) and 10\% heat inactivated fetal calf serum (ATCC-Cat. No. 30-2020), and cultured at $37^{\circ} \mathrm{C} / 5 \% \mathrm{CO}_{2}$ in a humid environment. Cells were sub-cultured when $80-85 \%$ confluency and only early passage cells were used for MN assays. One day prior to assay, MDCK cells were sub-cultured into flatbottomed 96 well plates at $2 \times 10^{4}$ cells/well in $100 \mu \mathrm{L}$. Serum samples were treated overnight with 3 volumes of RDE for $18 \mathrm{~h}$ at $37^{\circ} \mathrm{C}$, and then inactivated at $56^{\circ} \mathrm{C}$ for $30 \mathrm{~min}$. Plasma were then diluted 1:10 in virus growth media, comprising serum-free EMEM containing $0.6 \%$ BSA and $1 \mu \mathrm{g} / \mathrm{ml} \mathrm{N}$-p-Tosyl-Lphenylalanine chloromethyl ketone (TPCK)-treated trypsin (Worthington Biochemical), and two-fold serially diluted in virus growth medium in a separate 96-well plate. Reassortant H5N1 virus (NIBSC; Cat. 
No. NIBRG-14) was diluted to $100 \mathrm{TCID}_{50}$ per $50 \mu \mathrm{l}$ in virus growth media, and $50 \mu \mathrm{l}$ of virus added to wells containing the serially diluted plasma, and incubated for $1 \mathrm{~h}$ at $37^{\circ} \mathrm{C} / 5 \% \mathrm{CO}_{2}$. Control wells contained virus only and growth media only. After $1 \mathrm{hr}$ incubation, media from cell monolayers was replaced with the serum-virus mixtures and plates incubated for $1 \mathrm{hr}$. Serum-virus mixtures were then replaced with $200 \mu \mathrm{L}$ of virus growth media $+2 \%$ fetal bovine serum, and plates incubated for $48 \mathrm{~h}$ at $37^{\circ} \mathrm{C}$. Cells were then fixed in 4\% paraformaldehyde (Fisher Scientific; Cat. No. AAJ19943K2) in PBS for 30 min, washed in PBS and permeabilized in $0.1 \%$ PBS/Triton X-100 at RT for 15 min. Cells were then washed and blocked in a blocking buffer of $3 \%$ bovine serum albumen (BSA; Sigma-Aldrich, Cat. No. A9085) in PBS for $1 \mathrm{~h}$ at RT. Influenza virus nucleoprotein (NP) was then detected using anti-NP mAbs (Millipore Cat Nos. MAB 8257 and $M A B$ 8258) combined at 1/1000 dilution each in blocking buffer, followed by horse radish peroxidase (HRP)-conjugated anti-mouse IgG (KPL, Cat. No. 074-1802) diluted to 1:3000 in blocking buffer. Plates were developed in 3,3',5,5'-tetramethylbenzidine (TMB) peroxidase substrate (SureBlue ${ }^{\mathrm{TM}}$; SeraCare KPL Cat. No. 5120-0075) and reactions stopped using $0.18 \mathrm{M} \mathrm{H}_{2} \mathrm{SO}_{4}$. Assays were quantified in an enzymelinked immunosorbent assay (ELISA) plate reader (Emax Plus, Molecular Devices LLC, San Jose, CA) at 450nm using SoftMax® Pro 7.1 software.

2.7 T cell recall assays. Mice that had been immunized with trimerized H5 VN04 for serological studies were re-used for $\mathrm{T}$ cell recall assays at the serological endpoint. Prior to the assay, mice were boosted via the i.p. route with $100 \mathrm{~mL}$ the same vaccine formulation used to prime, and splenocytes were harvested 10 days later. Recall assays were performed using ELISpot format essentially as described previously (29). Antigens used for recall were HAs expressed in HEK293 cells (Sino Biological: H1 Cal09, Cat. No. 11055V08H; H5 VN04 Cat. No. 11062-V08H2; and H7 AH13 Cat. No. 40103-V08H) as well as Ebola virus glycoprotein expressed in insect cells (IBT BioServices Cat. No. 0501-015). Assays were performed in T cell medium (TCM) comprising Iscove's Modified Dulbecco's Medium (IMDM), containing $5 \times 10^{-5} \mathrm{M}$ bmercaptoethanol, $100 \mathrm{IU} / \mathrm{mL}$ penicillin, $100 \mathrm{mg} / \mathrm{ml}$ streptomycin, and $10 \%$ fetal calf serum as described (29). After $18 \mathrm{hrs}$ of incubation, the assay supernatants were collected for multiplex cytokine screening using the LEGENDplex ${ }^{\mathrm{TM}}$ kit (BioLegend Inc., San Diego, CA) according to the manufacturer's instructions, before the ELISpot was processed. T cell recall assays were also performed using virusinfected antigen-presenting cells ( $v$-APCs) as a source of as source of recall antigen for restimulating CD8 $T$ cells. For this, erythrocyte-depleted splenocytes were washed $3 x$ in PBS, then incubated with H5N1/PR8 reassortant influenza virus to a final concentration of $1 \mathrm{HAU}$ in serum-free infection medium (Eagles Minimal Essential Medium, 0.6\% BSA, $1 \mathrm{mg} / \mathrm{ml}$ EDCP-treated trypsin + antibiotics) at $37^{\circ} \mathrm{C} / 5 \% \mathrm{CO}_{2}$ with gentle agitation every 10 minutes. After $1 \mathrm{~h}$ incubation, v-APCs were washed $1 \mathrm{x}$ in PBS then resuspended at $10^{7}$ cells $/ \mathrm{ml}$ in TCM (as described above) containing $0.2 \%$ FCS. The v-APCs were used neat $\left(10^{7}\right.$ cells $/ \mathrm{ml}$ ) or diluted to $10^{6}, 10^{5}$ and 0 cells $/ \mathrm{ml}$ in a suspension of $10^{7}$ cells $/ \mathrm{ml}$ uninfected splenocytes in TCM. The titrated VAPCs were then mixed with an equal volume of responder splenocytes from either immunized or naïve control mice at $10^{7}$ cells $/ \mathrm{mL}$, and $100 \mathrm{~mL}$ of APC:responder cell suspension added to wells of pre-coated ELISPOT plates, or flat-bottomed 96 well plates for intracellular cytokine staining (ICS). Plates were incubated for $18 \mathrm{~h}$ before processing for cytokine detection by ELISpot, ICS, or supernatant assay by LEGENDplex ${ }^{\mathrm{Tm}}$. 
2.8 Intracellular cytokine staining_(ICS). Intracellular cytokine staining was performed using reagents according to the manufacturer's instructions (Biolegend Inc.). Briefly, cells were harvested from recall assays in round-bottom 96 -well plates by centrifuging at $400 \mathrm{x} \mathrm{g}$ for $5 \mathrm{~min}$, and surface-labeling the cells with an antibody cocktail comprising BV510-anti-CD8, BV650-anti-CD19, BV785-anti-CD4 and 7AAD for 20 mins at RT. After washing in Dulbecco's PBS (DPBS), cells were fixed and permeabilized for 20 mins at RT in Cyto-Fast ${ }^{\text {TM }}$ Fix/Perm buffer. After washing $2 x$ in Cyto-Fast ${ }^{\text {TM }}$ Perm Wash solution, cells were incubated with an intracellular antibody cocktail comprising PacBlue-anti-Tbet, AF488-anti-IFNg, PE-anti-Gata3, PECy7-anti-IL4, AF647-anti-IL17A and APCCy7-anti-IL2 in $50 \mathrm{~mL}$ per well of Cyto-Fast ${ }^{\text {TM }}$ Perm Wash for 30 mins at RT in the dark. After washing with $200 \mathrm{~mL}$ of Cyto-Fast ${ }^{\text {TM }}$ Perm Wash solution, cells were washed $2 x$ in Cell Staining Buffer (BioLegend Cat. No. 420201) and 100mL samples were acquired from 96-well plates on a Novocyte 3000 for $50 \mathrm{sec}$, with FSC-H threshold set at 100,000, and voltage settings such that unstained cells have autofluorescence values between $10^{2}-10^{3}$ units. Unstained and single-color stained controls were used to set up the compensation matrix. Flowjo software (BD Inc.) was used for flow cytometry compensation, additional data processing, gating, analysis and rendering figures. Briefly, live lymphocytes were gated by appropriate forward vs side scattering, followed by singlet gating by area vs. height of forward scattering pulse, and followed by 7AAD-gating of live (non-apoptotic and non-necrotic cells). Finally, analysis of each $\mathrm{T}$ cell subpopulation was conducted as summarized in the Results section.

2.9 B cell labelling. HA proteins (Sinobiological Inc.) were conjugated to fluorescent dyes using the DyLight $^{\text {TM }}$ Microscale Antibody Labeling Kit (Thermo Fisher Scientific, Waltham, MA; Cat. No. 22858) according to the manufacturer's instructions, as described previously (25). Briefly, lyophilized HAs were reconstituted in $100 \mathrm{~mL} \mathrm{H}_{2} \mathrm{O}(1 \mathrm{mg} / \mathrm{ml})$ and $8 \mathrm{~mL}$ of $0.67 \mathrm{M}$ borate buffer added. $\mathrm{H} 1, \mathrm{H} 5$ and $\mathrm{H} 7$ were added to DyLight ${ }^{\text {TM }} 405,488$ and 650 (Cat. Nos. 53021, 53025, 8543), respectively, vortexed gently and incubated at RT for $1 \mathrm{~h}$. Excess unbound dye was removed by gentle mixing with $108 \mathrm{~mL}$ of Dye Removal Resin (centrifuged prior to mixing to remove the storage buffer) followed by centrifugation at $1000 \times \mathrm{g}$ to collect the labelled proteins. For B cell labelling, splenocyte cell suspensions were first surface labelled at RT for 30 mins with a cocktail of fluorescent Abs and reagents, comprising BV510-anti-CD138, BV655-anti-CD3, BV785-anti-CD19, PE-anti-IgG2a, PE/Cy-anti-GL7, and APC/Cy7-anti-IgM (BioLegend Inc., San Diego, CA) and 7- aminoactinomycin D (7AAD; Thermo Fisher) at $2 \times 10^{6}$ cells/well in 96-well round-bottom plates. After washing in PBS, cells were fixed, permeabilized and washed using an intracellular staining kit (CytoFast $^{\text {tM }}$ from BioLegend) according to the manufacturer's instructions. Cells were then incubated in $100 \mathrm{ng} /$ well $(50 \mathrm{~mL}$ total vol) of fluorescently-labeled HA monomers in Cyto-Fast Perm/Wash buffer for $1 \mathrm{~h}$ then washed in Perm/Wash buffer. Data were acquired using a Novocyte 3000 flow cytometer for $50 \mathrm{sec}$, with FSC-H threshold set at 100,000, and voltage settings such that unstained cells have autofluorescence values between $10^{2}-10^{3}$ units. Unstained and single-color stained controls were used to set up the compensation matrix. Data were analyzed with FlowJo software. CD19+ (including dim or lo and bright or high) cells were gated in a two-dimensional CD19 vs. CD3 plot, followed by gating of antigen-specific (plasma) cells in a 2D CD138 vs. CD19 plot (plasma cells are defined CD138+ CD19lo). For additional 2D plots, gated plasma cells were plotted in 2D showing $\mathrm{H} 1$ vs. $\mathrm{H} 5, \mathrm{H} 1$ vs. $\mathrm{H} 7$ and $\mathrm{H} 5$ vs. $\mathrm{H} 7$ to measure 
potential cross-reactivity. Additional statistics were carried out using tables and layouts in FlowJo followed by rendering graphs in Excel (Microsoft Corp., Redmond, WA).

2.10 Single cell transcriptomic sequencing and analysis. Three C57BI/ 6 female mice were administered $5 \mathrm{mg} \mathrm{H} 5$ monomer (Sinobiological Cat. 11062-V08H1) in 50mL adjuvant (IVAX-1) comprising an equal mixture of AddaVAX emulsion (Invivogen) and PBS containing MPLA in liposomes and CpG ODN 1018, at final concentrations of 3 and 1 nmole, respectively. The formulation was administered the via the s.c. route (base of tail) and boosted similarly on d14, and again on d28 (second boost via i.p. route), and spleen (SP) and lymph nodes (LN) from multiple sites harvested 7 days later. Single cell suspensions of LN cells and erythrocyte-depleted SP were prepared by gently pressing tissues through a cell strainer. SP and $L N$ cell suspensions from two age-matched naïve mice were also prepared for comparison. The four samples (unimmunized LN, unimmunized SP, immunized LN, immunized SP) were then bar coded separately using cell multiplexing kits ( $3^{\prime}$ CellPlex Set A, 10 X Genomics, Cat. No. 1000261) at $2 \times 10^{6}$ total cells per sample following manufacturer's protocol. The final barcoded cells were resuspended at $\sim 2,000$ cell $/ \mathrm{mL}$, and then mixed to form two pools: one comprised unimmunized $L N$ and SP cells $(\sim 5,000$ each) in one droplet encapsulation and cDNA library preparation channel preparation channel, and the other immunized LN and SP ( 5,000 cells each) similarly prepared in another channel. These two channels were subjected to single-cell cDNA library preparation (10x Genomics Chromium 3' v3 kit) and then sequenced with a target depth of $\sim 50 \mathrm{~K}$ reads per cell using the Illumina HiSeq system. For read alignment and data preprocessing, FastQC (30) was first used to quality control FastQ format raw data using the fastqc command and the --noextract flag. The multiplexed samples were then demultiplexed, and the FastQs were aligned to the CellRanger (31) reference mm10-3.0.0 using STAR implemented in CellRanger v6.0.2 with the multi command and default parameters. The output .mtx and feature barcode files from CellRanger were read into SCANPY v1.7.2 (32). Only the SP data were considered for downstream analyses presented here. The following operations were performed using SCANPY's built-in functions unless otherwise stated. The resulting count matrices were concatenated such that only genes that were expressed in both unimmunized and immunized mice (hereafter referred to as "batches") were included. Only cells that were confidently demultiplexed, that were expressing more than 200 genes, and genes that were expressed in more than 3 cells, were considered for the downstream analysis. The count data were log-normalized using the "Seurat" method $(33,34)$. For data batch-correction, the data were reduced to 50 dimensions using principal component analysis. The principal components were batch-corrected using harmonypy implemented in SCANPY with defaults. The k-nearest neighbor graph was constructed on the batch-corrected principal components with 100 neighbors and cosine distance metric. The resulting graph was reduced to 2 dimensions using UMAP for visualization. The cell types were identified using a support vector machine (SVM) model implemented in scikit-learn v0.24.2 (35) with a sigmoid kernel and C=10 trained on unpublished single cell transcriptomic data. The features (genes) were subset to those that were present in both datasets, the features in the training dataset were fit and scaled using scikit-learn's StandardScaler function, and the features in the splenocyte dataset were scaled with the same scaler; $20 \%$ of the training data were held out for validation. Genes that were differentially expressed between different cell types were identified using the Mann-Whitney-U rank-sum test, with Benjamini-Hochberg 
correction (36). Ribosomal genes (Rps and Rpl) were excluded from the testing. Only genes with an absolute value of $\log _{2}$ fold change $>0.5$ were considered. Scrublet (37) v0.2.3 was used to determine doublet probabilities. Scrublet pairs random transcriptomes together to form a training dataset, which it then uses to train a k-nearest neighbors doublet classifier. The scrublet pipeline was run as specified by the documentation with defaults.

Cell-cell communication inferences were made using CellChat v1.1.2, a recently developed tool that generates and plots cell-cell communication probabilities and interaction strengths from single cell transcriptomic data (38). The normalized data were exported from SCANPY and imported into Seurat v4.0.3 using custom functions. The CellChat interaction strengths were generated for each dataset individually (with min.cells $=100$, all other parameters were left as defaults) and then combined. The differential interaction strength was plotted using the netVisual_diffInteraction function in CellChat with defaults. The comparison between all unvaccinated and vaccinated cells was performed by subsetting the treatment naïve and vaccinated groups until each celltype had the same number of cells in each group, then doing differential expression as above.

2.11 Other data analysis and statistics Protein microarray data were tested for significance between groups using two- tailed Mann-Whitney tests for unpaired data or two-tailed Kruskal-Wallis tests with Dunn's multiple-comparisons using GraphPad Prism 6.7 (GraphPad, La Jolla, CA, USA). A P value of $<0.05$ was considered statistically significant. Flow data were analyzed with FlowJo software (FlowJo Llc., Ashland, OR).

\section{Results}

3.1 Breadth and magnitude of IgG response is influenced by TLR agonist(s) and emulsion. Groups of C57BI/ 6 female mice (7-10 weeks old) were administered via s.c. route (base of tail) a single dose of trimerized VN04 H5 in a different TLR agonist (TLRa), or combination TLRa, and formulated without or with squalene oil-in-water emulsion (AddaVAX) as shown in Table 1. Agonists comprised Pam2CSK4 (TLR2/6a), pyrimido-indole derivative and monophosphoryl lipid A (both TLR4a), imidazoquinoline derivative (TLR7a), ODN 1018 CpG DNA containing a phosphorothioate backbone (TLR9a), and a chemically linked tri-agonist (TLR2/6_4_7) as described previously (23). The pyrimido-indole TLR4a is less potent than the conventional MPLA agonist for TLR4, but is more suited for tri-agonist synthesis $(23,39)$. Plasma samples were obtained at days 10, 28, 43 and 103 for probing against an influenza protein microarray described previously (25), which displayed 125 different monomeric full length HA (HAO) drift variants and $131 \mathrm{HA} 1$ variants, spanning all $18 \mathrm{HA}$ subtypes. Other than transient weight loss in the first $24 \mathrm{~h}$ after vaccination, the animals in each group showed normal increases in body weight (Supplementary Fig. S1).

IgG responses peaked $\sim \mathrm{d} 28-\mathrm{d} 43$; the IgG profiles by protein microarray for full-length HAO and HA1 on d28 are shown in Figs. 1 and Fig. 2, respectively. Full-length $\mathrm{H} 5$ drift variants on the array range overall from 90.3 - 99.8 (average 96.4) \% sequence identity with the immunizing HA (VNO4 H5), while drift variants of 
$\mathrm{H} 1$ (the closest phylogenetic group to $\mathrm{H} 5$ ) range from 59.5 - 65.6 (average 62.9) \% sequence identity with VN04 H5. These values are higher if the conserved HA2 regions are compared. Thus, $92.9-99.6$ (average 97.8) \% identity for $\mathrm{H} 5$ variants, and 75.7 - 77.9 (average 77.1) percent identity for $\mathrm{H} 1$ variants. In addition to $\mathrm{H} 1, \mathrm{H} 5$ immunization also induces heterosubtypic cross-reactivity for other closely-related Group $1 \mathrm{HAs}$, including $\mathrm{H} 2$ and $\mathrm{H} 6$. In the absence of AddaVAX emulsion (orange symbols in Figs. 1 and 2), the strongest responses were obtained with MPLA and the CpG/MPLA combination adjuvant (Figs. $1 G$ and $\boldsymbol{H}$ ). The linked TLR 2/6_4_7 tri-agonist and TLR 2/6a also induced robust H5 responses (Figs. $1 \mathrm{~A}$ and C), while unlinked TLR2/6,4,7 tri-agonist, TLR7a, and TLR9a induced weak, but measurable homosubtypic IgG cross-reactivity only without emulsion (Figs. 1B, E and F, respectively). H5 with pyrimido-indole derivative (TLR4a), or administered alone without TLRa, failed to induce detectable IgG under the conditions used (Figs. 1D and I, respectively). AddaVAX alone was an excellent adjuvant for H5 (Fig. 1I), and consistently elevated the responses induced by different TLR agonists. The largest effects on antibody responses by addition of AddaVAX (AddaVAX deltas) were seen when the response to TLR agonists alone was low, namely the unlinked TLR2/6,4,7 tri-agonist (Fig. 1B) and TLR4, TLR7, and TLR9 agonists (Figs. 1D, E and F, respectively). Of particular note, the pyrimido-indole derivative (TLR4 a), which failed to elicit detectable IgG against $\mathrm{H} 5$, produced robust homo- and hetero-subtypic cross-reactivity when co-administered with AddaVAX. This was higher than achieved with AddaVAX or TLR4a alone, indicative of a synergistic response. AddaVAX deltas were smaller where the TLR agonists alone induced responses, i.e., linked TLR2/6_4_7 tri-agonist (Fig. 1A), and TLR2/6, TLR4 and TLR9/4 agonists (Figs. 1C, $\mathbf{G}$ and $\mathbf{H}$, respectively). Nevertheless, the effect of the emulsion was often dramatic. For example, the cross-reactive response to $\mathrm{H} 5$ administered with unlinked tri-agonist, TLR7a, or TLR9a, were modest and homosubtypic only, whereas when the same agonists were co-administered in AddaVAX induced near maximal homosubtypic and heterosubtypic signals. This enhancement of heterosubtypic cross-reactivity by AddaVAX could potentially increase the utility of the vaccine to provide protection against novel subtypes with pandemic potential. Interestingly, the smallest AddaVAX delta was seen with MPLA (a TLR4a), which is by itself a strong adjuvant for $\mathrm{H} 5$; the profile after coadministration with AddaVAX was essentially unchanged. However, this may be related to the liposome needed for delivery of hydrophobic MPLA which may not benefit from an oil-in-water emulsion in the same way as other water-soluble agonists.

In addition to full-length HA, the protein microarray also displays HA1 fragments, which contain the variable head domain and part of the more conserved stalk of the protein. Previous studies with this array format have shown that sera with reactivity to the full-length (HA0) may, or may not, also recognize HA1, depending on whether there are antibodies present against the head $(25,40)$. IgG profiles to HA1 fragments are shown in Fig. 2. The TLR9a/4a (CpG/MPLA) combination adjuvant in AddaVAX was the only formulation able to engender robust IgG homosubtypic cross-reactivity for HA1 fragments of H5 (Fig. 2H). Modest responses were also generated using the individual TLR9a and TLR4a (MPLA) agonists alone when administered with emulsion (Figs. $2 \mathrm{~F}$ and $\mathbf{G}$ ) although the $\mathrm{IgG}$ signals when combined (Fig. $2 \mathrm{H})$ were greater than the sum when used alone. Of the other adjuvants tested, only the unlinked combination of TLR2/6a, TLR4a and TLR7a in the presence of emulsion (Fig. 2B) were able to induce lgG. The weaker response to HA1 compared to full length HAO may reflect the smaller size of the HA1 polypeptide, and consequently the fewer B cell epitopes available for recognition by the polyclonal 
response. Alternatively, the conformation of HA1 may not accurately reflect the conformation of the same sequence within the trimerized full-length protein, which may also contribute to the reduced strength of the response. To this point, we presented data previously (25) showing that conformation-sensitive mAbs raised against $\mathrm{H} 1$ from the 2009 pandemic recognized both $\mathrm{HAO}$ and $\mathrm{HA} 1$ on the array, suggesting the HA1 is folded correctly. Combination adjuvant CpG/MPLA/AddaVAX was the only adjuvant to engender detectable hetero-subtypic cross-reactivity, albeit against only one $\mathrm{H} 1$ and one $\mathrm{H} 2$ variant (Fig. $2 \mathrm{H}$ ). None of the other adjuvants tested were able to stimulate heterosubtypic cross-reactivity for HA1 fragments.

$\mathrm{H} 5$ has undergone antigenic drift in poultry and natural wildfowl populations, and different H5 variants are typically grouped into clades and subclades (41). A successful pre-pandemic vaccine will need to provide broad coverage across different clades to provide anticipatory protection. Thus, Fig. $\mathbf{3}$ shows array data of $\mathrm{H} 5$ variants by clade, which reveals broad cross-clade reactivity generated by the majority of adjuvants tested. In the absence of AddaVAX, cross-clade reactivity is the strongest in TLR9a/TLR4a, (CpG/MPLA), TLR 4a (MPLA), linked TLR 2/6, 4_7a, and TLR 2/6 adjuvant groups. In the presence of Addavax, crossclade reactivity is elevated for all adjuvant groups.

In addition to antibody profiles, we assessed the dynamics of the response. Fig. $\mathbf{4}$ shows the development of homosubtypic reactivity at 3 time points after a single dose. In the absence of AddaVAX, the TLR9aTLR4a (CpG/MPLA) combination adjuvant produced the most rapid response, being the only vaccine to have elicited detectable antibodies on day 10 . By day 28 , IgG reactivity was also seen from linked TLR 2/6_4_7a tri-agonist, TLR2/6a, and TLR4a (MPLA), which remained elevated at day 42. In the presence of AddaVAX, all the vaccines elicited reactivity by day 28 , although CpG/MPLA/AddaVAX again elicited the most rapid response, as can be seen on day 10. We conclude from these data, and data presented in Fig. 1 and 2, that of all the adjuvants tested, CpG/MPLA/AddaVAX induces the most rapid response, with the greatest magnitude and broadest homo- and hetero-subtypic cross-reactivity.

3.2 Th1/Th2 balance is variable according to adjuvant used. Both IgG1 v IgG2c subtyping on microarrays and cytokine profiling in T cell recall assays were performed on the same groups of immunized $\mathrm{C} 57 \mathrm{BI} / 6$ mice to evaluate the Th1/Th2 response elicited by each adjuvant tested (Fig. 5). In the absence of AddaVAX emulsion, TLR2/6_4_7a tri-agonist, and individual TLR2/6a and TLR7a stimulated IgG1 antibodies (Th2) (Fig. 5A), while TLR9a was strongly polarized to IgG2c (Th1), and the combination adjuvant TLR9a/TLR4a (CpG/MPLA) gave a more balanced response. Overall, the addition of AddaVAX elevated the $\lg \mathrm{G} 1$ and IgG2c signals but did not reverse any polarization induced by the TLR agonists alone. Thus, AddaVAX elevated the IgG1 signals to both linked and unlinked 2/6_4_7 tri-agonists, as well as individual TLR 2/6, 4 and 7 agonists. Responses to TLR9a (CpG) and TLR4a (MPLA) and the combination TLR9/4a (CpG/MPLA) were more balanced with both IgG1 and IgG2c detected. It is noteworthy that in the absence of AddaVAX responses to some TLR agonists were undetectable (i.e., unlinked tri-agonist, and individual TLR4 (PID) and TLR9 agonists); however, in the presence of AddaVAX all three were strongly immunogenic. Finally, we noted H5 antigen with AddaVAX alone (i.e., without TLR agonists) was also balanced. 
IFN-g and IL-4 release was determined from the same mice at the experimental endpoint by ELISpots (Fig. 5B). The recall assay included immunizing (H5) antigen, as well as hemagglutinins $\mathrm{H} 1$ and $\mathrm{H} 7(63 \%$ and $42 \%$ sequence identity with $\mathrm{H} 5$, respectively). Despite observing heterosubtypic cross-reactivity at the $\mathrm{Ab}$ level, IFN-g and IL-4 spot-forming cells (SFCs) were detected against H5 only. Several cytokine patterns were consistent with IgG1/lgG2c profiles. For example, in the absence of AddaVAX, the only two groups to induce IgG2c were agonists to TLR4 (MPLA) and the combination TLR9/4a (CpG + MPLA) adjuvant; accordingly, these also produced H5-specific IFN-g SFCs in the recall assay. In the presence of AddaVAX, these two agonists elicited elevated signals to IgG1 which correlated with the presence of IL-4 SFCs. Similarly, in the absence of AddaVAX, linked TLR 2/6_4_7 tri-agonist and TLR 2/6 and 7 agonists produced only IgG1 (Th2) and no IFN-g SFCs, of which two (linked TLR tri-agonist and TLR 2/6 agonist) produced detectable IL-4 SFCs. In the presence of AddaVAX, linked and unlinked TLR tri-agonist, and individual TLR 2/6, 4 and 7 agonists produced strongly polarized IgG1 (Th2) responses and correspondingly low numbers of IFN-g SFCs but detectable IL-4. This contrasts with AddaVAX alone, which produces a balanced IgG1/lgG2c profile, suggesting these TLR agonists suppress the production of IgG2c. Of note is the TLR4a, PID, which appears ineffective at inducing antibody or cytokines in the absence of AddaVAX, but which becomes a strong Th2-polarizing adjuvant in the presence of AddaVAX, displaying strong IgG1 signals and robust IL-4 SFC numbers. Note this contrasts with the combination TLR 9/4a $(C p G+M P L A)$ in the absence of emulsion, which strongly polarizes the response to $\operatorname{lgG} 2 \mathrm{c}(\mathrm{Th} 1)$ and a production of IFN-g SFCs. Thus, the breadth of the cross-reactive response and polarization can be tuned according to the adjuvant, as summarized in Table 2.

The largest numbers of IFN-g and IL-4 producing SFCs was induced by the combination of MPLA, CpG and AddaVAX. This appears to result from synergy between AddaVAX and either TLR agonist, as the SFC numbers for MPLA+AddaVAX or CpG+AddaVAX were greater than the sum of SFC numbers for the individual components. Measurements of antigen-specific $B$ cells also suggest these components are synergistic (see section 3.3 below).

Additional cytokines were assayed in the supernatants of recall assay using multiplex bead detection (Fig. 5C). The highest concentrations were elicited by MPLA/AddaVAX and CpG/MPLA/AddaVAX combination adjuvants. The former produced high levels of Th1-associated IL-2 and TNF-a, as well as high levels of IL6 , which is produced by different APCs and promotes Th2 differentiation (42). These data are consistent with the balanced IgG1/lgG2c profile seen by arrays and ELISPOTs. CpG/MPLA/AddaVAX also produced high levels of IL-2 and TNF-a, but lower levels of IL-6, which may contribute to the skewing toward IgG2C over IgG1. Both MPLA/AddaVAX and CpG/MPLA/AddaVAX also induced modest levels of Th17associated cytokines. Among the non-emulsified formulations (left side of Fig. 5), of note is CpG/MPLA. This adjuvant is strongly biased to Th1 with elevated IL-2 and TNF-a but low IL-5 and IL-6, consistent with the strongly polarized IgG2C response. MPLA produces a more balanced Th1/Th2 cytokine profile, consistent with the balanced IgG1/lgG2c response. The remaining non-emulsified agonists, which produced mainly IgG1 skewed responses, produced both Th1 and Th2-associated cytokines. Interestingly, H5 alone (without TLRa or emulsion), which did not induce detectable antibodies, was associated with the release of several cytokines in the recall assay. This profile was amplified by AddaVAX, notably release of 
IL-5. This would suggest $\mathrm{H} 5$ has an inherent capacity to stimulate a $\mathrm{T}$ cell response, as has been reported by others $(43,44)$, although the mechanism is unknown.

3.3 CpG and MPLA synergize in the induction of antigen-specific plasma cells. It was of interest to determine whether the effects of combining $\mathrm{CpG}$ and MPLA in AddaVAX were synergistic, since synergies may allow lower doses of adjuvant to be used and development of less reactogenic vaccines. To address this, we used antigen-specific B cell labelling to quantify these in mice administered influenza HA in CpG/MPLA/AddaVAX, and compared these with CpG/AddaVAX or MPLA/AddaVAX individually. The CpG/MPLA/AddaVAX combination adjuvant induced more total plasma cells (Fig. 6A) and antigenspecific B cells (Fig. 6B) than the sum of CpG/AddaVAX and MPLA/AddaVAX separately, indicating a synergistic effect. Almost all the antigen-specific $B$ cells were $C D 138+$, indicating their differentiation into plasma cells. These numbers are shown as bar charts in Fig 6C. Ig isotype staining of antigen-specific B cells revealed both IgG1 and IgG2a are produced (Fig. 6D), although the majority expressed IgG2a, consistent with the Th1>Th2 polarization seen by array profiling, and T cell recall assay (Fig. 5).

3.4 Further characterization of the utility of IVAX-1 as an adjuvant. Overall, the data presented thus far show that CpG, MPLA and AddaVAX, a combination adjuvant we term 'IVAX-1', synergize to induce the most rapid and broadest cross-reactive response among all the adjuvants tested. Therefore, we performed a series of experiments to characterize IVAX-1 further. Fig. 7A shows plasma generated using VN04 H5/IVAX-1 neutralized H5N1 reassortant virus in vitro. We noticed, as reported previously (25), that boosting was required to reach detectable levels of neutralization. We showed earlier that IVAX-1 engendered a robust IFNg-response in the ELISpot (Fig. 5B). As the antigen for recall was supplied as soluble $\mathrm{H} 5$, it was expected that most of the processed antigen would be presented in the context of class II MHC to CD4 T cells, rather than to CD8 T cells. This was confirmed when intracellular cytokine staining (ICS) was used in place of the ELISpot to identify the source of IFNg (Fig. 7B). Therefore, we attempted to improve the recall of CD8 T cells using H5N1 influenza virus-infected splenocytes (vAPCs). However, we saw no improvement in detection of antigen-specific CD8 cells. Although the data support the notion that IVAX-1 preferentially stimulates CD4 T cells, we cannot exclude a CD8 T cell response as infected respiratory epithelial cells (the natural host cell for IAV) may be required for vAPCs instead of splenocytes. Finally, we explored intranasal (i.n.) administration of IVAX-1, since the above experiments were based on the s.c. route only. The i.n. route is often used to engender an IgA response in the nasal and respiratory mucosa. Mice were administered H5/IVAX-1 via the i.n. route and boosted on d56. Other than transient weight loss $(<8 \%)$ within $24 \mathrm{~h}$ of the prime and boost, there were no adverse events associated with this intranasal delivery or boosting (not shown). At the experimental endpoint (d69), IgG, IgG1, IgG2c and IgA profiles were determined for plasma, and nasal and lung washed by microarray (Fig. 7C). Robust IgG responses were seen in plasma, dominated by subtype IgG2c, consistent with the findings seen when the same vaccine was administered by the s.c. route, as shown in Fig. 5. Robust IgA responses were found in nasal and lung washes, and IgG was also found in the lung, although no IgA was detected in plasma. Ab profiles recognized by $\lg G, \lg G 2 \mathrm{c}$ and $\lg A$ were identical after i.n. administration, and comprised recognition of both $\mathrm{H} 5$ (homo-subtypic) and $\mathrm{H} 1$ (hetero-subtypic) variants. Indeed, the IgG profile induced by i.n. administration was highly correlated with that induced by s.c. administration (Fig. 7D). 
3.5 Single-cell mRNA sequencing_(sc mRNA seq) analysis. Finally, in order to generate a transcriptomic "fingerprint" of IVAX-1 for future comparison with other adjuvants, we performed an unbiased single cell transcriptomic analysis of whole spleen cell suspensions pooled from 3 immunized B6 mice that received H5/IVAX-1 barcoded using the 10x Genomics platform and sequenced on the Illumina HiSeq, and performed a similar analysis on an aged-matched naïve control B6 mouse for comparison (see methods). To our knowledge, this is the first such dataset generated from whole lymphoid tissue without prior cell sorting. A previously trained supervised support vector machine model (SVM) was first used to define different cell types based on transcriptional profiles. The majority of splenocytes were of lymphoid origin with some myeloid cells, as shown in the Uniform Manifold Approximation and Projection (UMAP) plot in Fig. 8A and Supplemental Fig S2A. The heatmap in Fig. 8B shows expression profiles of transcriptionally active splenocytes in mice receiving H5/IVAX-1. Cell populations were identified by differential gene expression of the subtype compared to all other subtypes. Overall, three main arms are delineated: $T$ lymphocytes (which also includes NK/T cells, gd T cells, and Tregs), B lymphocytes, and myeloid cells (myeloid dendritic cells, macrophages, monocytes and neutrophils). Interestingly, the unbiased hierarchical clustering of cell type transcription profiles placed B cells more closely related to myeloid cells than to $T$ cells. The major cell populations were well delineated by marker genes. Thus, the T cells cluster show elevated expression of Trac, Trbc2, Cd3g and Cd3d (TCR a and b chain constant domains, and CD3g and d chains, respectively). Others include Ms4a4a and $b$ (CD20 homolog expressed on mature Th1 cells (45)); Tcf7 (T cell-specific transcription factor (46)); and Skap1 (regulator of TCR signaling through MAP kinase, (47)). Nkg7 and Ctsw genes (NK cell granule protein 7 and cathepsin W) are particularly elevated in NK/T cells and play roles in cell-mediated killing by NK and CD8 T cells $(48,49)$. Also of note is expression of II2rb (IL2 receptor b chain) which is expressed in NK/T and regulatory T cells (Tregs) at this time point. Also expressed in Tregs is the signature gene Izumo1r (Folr4; folate receptor) $(50,51)$. The B cells arm shows elevated expression of $C d 79 a$ and $C d 79 b$ (BCR CD79a and b chains); Ebf1 (B cell-specific transcription factor (52)); Ighm (IgM heavy chain); and Cd74 (MHC class II invariant chain (53)). Cells in the myeloid cell express Fcer1g (IgE Fc receptor); Tyrobp (an immunoreceptor-associated tyroskine kinase); and Ifitm3 (IFN-induced transmembrane protein 3), an anti-viral protein that which restricts the entry of several viruses into host cells (54). Among the myeloid cells, neutrophils show expression of S100 family proteins, including S100a8 and S100a9 (myeloid-related proteins 8 and 14), which are known to be released by activated phagocytes and amplify the inflammatory effect of LPS mediated through TLR4 (55). Also expressed in neutrophils is Msrb1 (methionine reductase) which appears to enhance the expression of anti-inflammatory cytokines by macrophages (56), and $H p$ (haptoglobulin), a major acute phase protein. These data serve as an additional verification that the SVM correctly identified the cell types. Interestingly we found a small population of B and T cell doublets which were identified due to their expression of both T cell markers ( $\mathrm{Cd} 3 \mathrm{~g}$ ) and B cell markers (Cd79a) and an unbiased doublet detection algorithm (Supplementary Fig. S2B-D). Doublets in droplet-based sequencing platforms can be indicative of juxtacrine signaling.

We then asked whether the differential IgG2c>lgG1 response characterized above could be corroborated using the transcriptomic data. We found $l g h g 2 c, l g h g 2 b$ and $l g h g 1$ genes were all upregulated in B cells from immunized mice relative to naïve mice (Supplementary Fig. S3 and Fig. 8C), with log2-fold changes 
of $6.21(\mathrm{p}$-value $=2.14 * 10-93), 4.35\left(\mathrm{p}\right.$-value $\left.=2.33^{\star} 10-281\right)$ and $2.69(\mathrm{p}$-value $=1.10 * 10-158)$, respectively (Mann-Whitney $U$ rank-sum test). This is consistent with the moderately skewed IgG2c>lgG1 response observed by IgG isotyping after IVAX-1 administration (Fig. 5a). We also compared the expression of the Ifng gene in naïve and immunized mice (Fig. 8D), which showed IFNg was expressed by both CD4 and CD8 T cells, with log2-fold increases of 0.7 ( $p$-value $=0.28$ ) and 0.9 ( $p$-value $=0.69)$, respectively (MannWhitney $U$ rank-sum test), consistent with a robust IFNg response in T cell recall assays (Fig. 5B). Interestingly, intracellular cytokine staining suggested IVAX-1 was not a strong inducer of antigen-specific CD8 cells (Fig. 7B), although as mentioned above, this may reflect suboptimal restimulation conditions for CD8 cells in the recall assay. Overall, these data show that the transcriptomic Th1/Th2 balance matched that defined by IgG subtyping and cytokine expression in T cell recall assays.

We also inferred cell-cell signaling interactions between different cell types using CellChat, a recently developed tool that defines intercellular signaling strengths between specified cell types in single-cell transcriptomic data. This tool has previously been used to analyze differential wound healing signaling in adult mice (38). As shown in Fig. 8E, lymphocytes (i.e., NK/T cells, T cells, Tregs and B cells) communicate predominantly with macrophages before immunization, whereas this shifts to dendritic cells and neutrophils after immunization. The gd $T$ cells, $B / T$ cell doublets and resident monocytes were excluded from the analysis because they did not meet the cutoff of 100 cells. Intra-cell type communication (e.g., $T$ cells to $T$ cells) is higher for lymphocytes in unimmunized mice and higher for neutrophiles and dendritic cells in immunized mice. Overall, these data provide a useful first look at the transcriptomic and cellular interactome induced by IVAX-1 at a single time point. Similar studies at different time points after vaccination, and with different adjuvants for comparison are in progress.

\section{Discussion}

In this study, we administered mice recombinant influenza virus hemagglutinin (HA) formulated with a panel of different adjuvants. A major aim was to identify adjuvants able to induce the broadest $A b$ response across different HA subtypes and drift variants using the microarray approach. Array profiling showed several of the tested adjuvants induced homo- and hetero-subtypic cross-reactivity profiles against full-length HA proteins. The response to IVAX-1 (CpG + MPLA + AddaVAX) in particular, was among the broadest and also the most rapid (Fig. 4), and unique among the adjuvants for eliciting homosubtypic responses to the HA1 portion (Fig. 3) that contains the variable head domain. IVAX-1 induced a balanced response comprised of both IgG1 and IgG2c secreting B cells, with a dominant Th1 cytokine profile in T cell recall assays (Fig. 5). A key observation was IVAX-1 induced higher numbers of antigen-specific plasmablasts than the sum of the numbers when $\mathrm{CpG} /$ AddaVAX or MPLA/AddaVAX were used separately (Fig. 6), suggesting a synergistic rather than additive effect. Unlike other adjuvants such as complete Freund's adjuvant or Titermax, IVAX-1 can be used for boosting without inducing lesions or other adverse events (data not shown). IVAX-1 can also be administered intranasally, and elicits IgA in lung and nasal washes (Fig. 7); the IgA profile displayed a cross-reactivity pattern that was identical to the IgG profile induced by the same route or by s.c. administration, suggesting the i.n. route could offer a needle-free, self-administrable alternative to i.m administration. Despite observing heterosubtypic cross- 
reactivity by antibodies, $\mathrm{T}$ cell responses in $\mathrm{B} 6$ mice administered $\mathrm{H} 5$ were $\mathrm{H} 5$-specific and did not show heterosubtypic cross-reactivity for $\mathrm{H} 1$ or $\mathrm{H} 7$. We speculate this is most likely related to a paucity of shared sequences in these HA subtypes capable of being presented by I-A ${ }^{b}$, the only class II MHC allele available in $\mathrm{C} 57 \mathrm{BI} / 6$ mice for presentation, although this has to be tested experimentally. Finally, we performed a preliminary sc mRNA seq study. In an unbiased analysis of the data, of note was elevated expression of lghg2c, lghg $2 b$ and lghg1 genes in immunized mice relative to naïve mice, consistent with the lgG subtyping by serology and flow cytometry.

The technology for producing influenza vaccines had changed little since the 1930's. Virus is propagated in fertilized chicken eggs and inactivated using propriolactone, followed by detergent extraction to enrich the lipid membrane fraction containing the integral HA and NA antigens (so-called 'split' vaccines). Tissue culture-derived (Flucelvax ${ }^{\circledR}$ ) and recombinant protein (Flublok ${ }^{\circledR}$ ) vaccines are widely available, these are mainly used as alternatives to egg-based vaccines in individuals with egg allergies. Most seasonal influenza vaccines are also administered without adjuvant, in part because inactivated virus vaccines retains some inherent immunogenicity $(57,58)$. Effectiveness is likely related to pre-existing immunity which is easier to boost than a primary response. Protection is short-lived, in part because bone marrow plasma cells quickly wane (59). FluAd® (Seqirus), currently the only adjuvanted seasonal vaccine, is a tetravalent inactivated split virus vaccine administered in MF59 (60), a squalene-in-water emulsion that is very similar to AddaVAX (61). The Ab response to FluAd® is broader than non-adjuvanted equivalents (60, $62,63)$. Yet, this vaccine is administered only to the elderly (>65 years), where its improved immunogenicity is used to overcome immune senescence rather than for increased breadth or other benefits. Adjuvants play an important role in pandemic preparedness by accelerating the response, enhancing immunogenicity and breadth, and dose-sparing of vaccine $(64,65)$. Early efforts at adjuvanting a pre-pandemic H5N1 vaccine using alum (aluminum salts such as aluminum hydroxide, which until 2017 was the only adjuvant available for human use) failed to elicit sufficient neutralization (66). However, the use of modern emulsions has led to the licensure of several pre-pandemic vaccines, including Q-Pan (ID Biomedical Corporation of Quebec), a monovalent split H5N1 vaccine, and Pandemrix (GlaxoSmithKline) monovalent split H1N1 vaccine, both adjuvanted with AS03 (GSK), a squalene-in-water emulsion with atocopherol (67).

A small but growing number of pattern recognition receptor (PRR) agonists are being used in licensed vaccines. These agonists represent a new generation of vaccine adjuvants, and can be classified according to the particular PRR signaling pathway they act through, i.e., a) toll-like receptor (TLRs), b) nucleotide-binding oligomerization domain-like (NOD) receptors, and c) stimulators of the cGMP-AMP Synthase STimulator of INterferon Genes (cGAS-STING) pathway. Adjuvant system 4 (AS04) from GSK comprises traditional alum combined with monophosphoryl lipid A (MPLA), a synthetic form of the TLR4binding domain of lipopolysaccharide (LPS) (68), is currently used with the approved vaccines against HPV and HBV, Cervarix (69) and Fendrix (70), respectively. Shingrix, an approved vaccine against shingles (herpes zoster virus) is adjuvanted with $A S 01_{B}$, which consists of MPLA and the natural saponin surfactant, Quil-A (QS-21) (71). Synthetic oligodeoxynucleotides containing unmethylated cytosineguanine dinucleotide motifs (ODN CpG) exerts adjuvant effect through TLR9 $(72,73)$ and has recently 
been approved as adjuvant for the recombinant HBV vaccine, Heplisav-B ${ }^{T M}(74,75)$. PRR agonists are yet to be approved for split inactivated seasonal influenza virus vaccines, despite studies showing there may be several benefits, including increasing magnitude, breadth and duration of the response. These benefits have been reviewed extensively in recent years $(64,76-78)$.

There is also increasing awareness that combining adjuvant components also increases the efficacy of vaccines though enhanced stimulation of APCs and lymphocytes (79-85). In this study, we saw a synergistic effect on Ag-specific plasmablast numbers when combining $\mathrm{CpG}$ and MPLA. Other studies have shown CpG + MPLA stimulate the induction of IL12 from dendritic cells, while neither agonist was effective when used alone (82). The same group also showed single-dose administration of an inactivated split influenza vaccine with $\mathrm{CPG}$ and MPLA led to improved homo- and hetero-subtypic cross-protection compared to vaccine administered with $\mathrm{CpG}$ or MPLA alone (83). Consistent with the findings reported here, CpG + MPLA induced IgG2c (Th1) polarization. The effects of CpG and MPLA alone were more discrepant; in our hands, $C p G$ failed to induce a significant $A b$ response after a single dose, and MPLA induced both isotypes (IgG2c>lgG1), while Ko et al (83) reported CpG induced IgG2c and MPLA induced $\lg \mathrm{G} 1$ (Th2). These discrepancies may have been caused by the different antigen preparations used (recombinant protein used here, vs. split inactivated virus) or the doses of antigen or TLRa administered. A general consensus is both CpG and MPLA engender a Th1-biased response in B6 mice and humans (86, 87). The molecular basis of CpG + MPLA synergy has been hypothesized to arise from activation of both MyD88 and TRIF signaling pathways $(88,89)$.

In this study we used recombinant HA proteins, rather than split inactivated virus, to evaluate different adjuvants. Recombinant proteins may offer several advantages over current egg-based methods, including more rapid manufacture, flexibility in design, and avoidance of adaptation to growth in eggs. HA stalk-focused immunization strategies to overcome antigenic drift are also readily amenable to recombinant protein approaches $(90)$. Currently, Flublok® is the only approved recombinant HA-based seasonal vaccine, comprised of HA proteins from four influenza viruses expressed in insect cells. Flublok is administered without adjuvant, although immunogenicity is achieved by using a 3-fold higher dose of each HA protein, i.e., $45 \mu \mathrm{g}$ compared to $\sim 15 \mu \mathrm{g}$ for a typical split vaccine (64). In our hands, doses of $2.5 \mathrm{mg} \mathrm{H} 5$ protein was unable to elicit an antibody response in $\mathrm{B} 6$ mice without adjuvant although high doses may overcome this. We also used a single dose regimen. Although $\mathrm{MN}$ and $\mathrm{HI}$ assays were performed on the plasma samples collected in the adjuvant screen herein, we saw no virus neutralization (data not shown), consistent with our previous studies (25) and other data (Fig. 7A) that show boosting is required to achieve virus neutralization, at least with the antigen doses used here.

Overall, the studies described here are consistent with the notion that adjuvanted recombinant HA proteins are a possible path toward broader vaccine coverage than is currently achieved with non-adjuvanted inactivated vaccines, particularly if used in conjunction with multivalent antigens (25). Achieving a true 'universal' vaccine will be challenging using adjuvants alone, without additional stalk directed immunization strategies to target conserved epitopes. Intuitively, cross-reactivity between variable epitopes is likely to be less effective than between conserved regions, as substitutions of just a few key 
amino acids in an epitope can abrogate neutralization. We speculate this may be offset by the use of adjuvants, which, by driving affinity maturation, may lead to higher affinity nAbs better able to tolerate substitutions than those generated by non-adjuvanted vaccines. This has the added benefit of exploiting the natural immunodominance of the head domain during vaccination and natural infection.

\section{Declarations}

\section{Acknowledgements}

We thank Quy Nguyen and Melanie Oakes, UCI Genomics High Throughput Facility, for single cell transcriptomic sequencing. We also thank the UCI Laser Spectroscopy Labs for use of dynamic light scattering facilities. Supported by Defense Threat Reduction Agency (DTRA) grant HDTRA1-18-1-0036 and NIH U01 grant Al160397. ED and QN were supported by the NSF grant DMS1763272 and a grant from Simons Foundation (594598). ED is also supported by the NIH grant T32GM136624. Work in the Krammer laboratory was supported by NIAID Collaborative Influenza Vaccine Innovation Centers (CIVIC) contract 75N93019C00051. The views expressed in this article are those of the authors and do not reflect the official policy or position of the U.S. Department of Defense or the U.S. Army.

\section{Author contributions.}

JEH-D, EJP, and DHD designed experiments; JEH-D, DHD, EJP, JF, SS, SJ, TJA, AJ, RN, and AJ, performed experiments; AE-K provided reagents; ED performed transcriptomic data analysis; FK, PLF, QN and DHD provided funding; JEH-D and DHD wrote the manuscript with input from all authors.

\section{Competing interests statement.}

JEH-D, JF, EJP, AJ, SJ, RN, AJ, PLF and DHD own shares in Nanommune Inc. Nanommune does not sell any products described in this paper, nor funded any part of the work described herein. Neither Nanommune or its shareholders are likely to benefit from the results described in this publication. The remaining authors declare that the research was conducted in the absence of any commercial or financial relationships that could be construed as a potential conflict of interest.

\section{References}

1. Bouvier, N. M. \& Palese, P. The biology of influenza viruses. Vaccine, 26S, D49-D43 (2008).

2. Kim, H., Webster, R. G. \& Webby, R. J. Influenza Virus: Dealing with a Drifting and Shifting Pathogen. Viral Immunol, 31, 174-183 (2018).

3. Altman, M. O., Angeletti, D. \& Yewdell, J. W. Antibody Immunodominance: The Key to Understanding Influenza Virus Antigenic Drift. Viral Immunol, 20, 362-372 (2018).

4. Petrova, V. N. \& Russell, C. A. The evolution of seasonal influenza viruses. Nat Rev Microbiol, 16, 4760 (2018).

5. Luo, M. Influenza virus entry. Adv Exp Med Biol, 726, 201-221 (2012). 
6. Wu, N. C. \& Wilson, I. A. Influenza hemagglutinin structures and antibody recognition. Cold Spring Harb Perspect Med, 10, a038778 (2020).

7. Ciminski, K., Pfaff, F., Beer, M. \& Schwemmle, M. Bats reveal the true power of influenza A virus adaptability. PLoS Pathog, 16, e1008384 (2020).

8. Wu, Y., Wu, Y., Tefsen, B., Shi, Y. \& Gao, G. F. Bat-derived influenza-like viruses H17N10 and H18N11. Trends Microbiol, 22, 183-191 (2014).

9. Wiley, D. C., Wilson, I. A. \& Skehel, J. J. Structural identification of the antibody-binding sites of Hong Kong influenza haemagglutinin and their involvement in antigenic variation., 289, 373-378 (1981).

10. Air, G. M., Laver, W. G. \& Webster, R. G. Mechanism of antigenic variation in an individual epitope on influenza virus N9 neuraminidase. J Virol1990/12/01, 64, 5797-5803 (1990).

11. Urbaniak, K. \& Markowska-Daniel, I. In vivo reassortment of influenza viruses (Acta Biochim Pol. Acta Biochimica Polonica, 2014).

12. Wan, X. F. Lessons from Emergence of A/Goose/Guangdong/1996-Like H5N1 Highly Pathogenic Avian Influenza Viruses and Recent Influenza Surveillance Efforts in Southern China. Zoonoses Public Health, 59, 32-42 (2011).

13. Cattoli, G. et al. Antigenic Drift in H5N1 Avian Influenza Virus in Poultry Is Driven by Mutations in Major Antigenic Sites of the Hemagglutinin Molecule Analogous to Those for Human Influenza Virus. $J$ Virol, 85, 8718-8724 (2011).

14. Sutton, T. C. The pandemic threat of emerging H5 and H7 avian influenza viruses. Viruses, 10, 461482 (2018).

15. Agor, J. K. \& Özaltın, O. Y. Models for predicting the evolution of influenza to inform vaccine strain selection. Hum Vaccines Immunother, 14, 678-683 (2018).

16. Sasaki, S. et al. Limited efficacy of inactivated influenza vaccine in elderly individuals is associated with decreased production of vaccine-specific antibodies. J Clin Invest, 121, 3109-3119 (2011).

17. Zbinden, D. \& Manuel, O. Influenza vaccination in immunocompromised patients: Efficacy and safety. Immunotherapy, 6, 131-139 (2014).

18. Crowe, J. E. Is It Possible to Develop a "Universal”Influenza Virus Vaccine? Potential for a Universal Influenza Vaccine

19. Angeletti, D. et al. Outflanking immunodominance to target subdominant broadly neutralizing epitopes (Proc Natl Acad Sci U S A, 2019).

20. Guthmiller, J. J. et al. First exposure to the pandemic H1N1 virus induced broadly neutralizing antibodies targeting hemagglutinin head epitopes. Sci Trans/ Med, 13, eabg4535 (2021).

21. Krammer, $F$. The human antibody response to influenza A virus infection and vaccination. Nat Rev Immunol, 19, 383-397 (2019).

22. Krammer, F. et al. A carboxy-terminal trimerization domain stabilizes conformational epitopes on the stalk domain of soluble recombinant Hemagglutinin substrates. PLoS One, 7, e43603 (2012). 
23. Albin, T. J. et al. 2019. Linked Toll-Like Receptor Triagonists Stimulate Distinct, CombinationDependent Innate Immune Responses.ACS Cent Sci5

24. Kilkenny, C., Browne, W. J., Cuthill, I. C., Emerson, M. \& Altman, D. G. 2010. Improving bioscience research reporting: The arrive guidelines for reporting animal research.PLoS Biol8

25. Hernandez-Davies, J. E. et al. Administration of Multivalent Influenza Virus Recombinant Hemagglutinin Vaccine in Combination-Adjuvant Elicits Broad Reactivity Beyond the Vaccine Components. Front Immunol, 12, 2695 (2021).

26. Liu, W. C., Nachbagauer, R. \& Florian Krammer, R. A. A. Assessment of Influenza Virus Hemagglutinin Stalk-Specific Antibody Responses. Methods Mol Biol, 1836, 487-511 (2018).

27. Gross, F. L., Bai, Y., Jefferson, S., Holiday, C. \& Levine, M. Z. Measuring influenza neutralizing antibody responses to $A(H 3 N 2)$ viruses in human sera by microneutralization assays using MDCK-SIAT1 cells. $J$ Vis Exp, 129, 56448 (2017).

28. Hobson, D., Curry, R. L., Beare, A. S. \& Ward-Gardner, A. The role of serum haemagglutination-inhibiting antibody in protection against challenge infection with influenza A2 and B viruses. J Hyg (Lond), 70, 767-77 (1972).

29. Davies, D. H. et al. T Cell Antigen Discovery Using Soluble Vaccinia Proteome Reveals Recognition of Antigens with Both Virion and Nonvirion Association (J Immunol, 2014).

30. Andrews, S. 2010. FastQC: A quality control tool for high throughput sequence data. https://www.bioinformatics.babraham.ac.uk/projects/fastqc/

31. Zheng, G. X. Y. et al. 2017. Massively parallel digital transcriptional profiling of single cells.Nat Commun8

32. Wolf, F. A., Angerer, P. \& Theis, F. J. SCANPY: large-scale single-cell gene expression data analysis. Genome Biol, 19, 15 (2018).

33. Satija, R., Farrell, J. A., Gennert, D., Schier, A. F. \& Regev, A. spatial reconstruction of single-cell gene expression data. Nat Biotechnol, 33, 495-502 (2015).

34. Stuart, T. et al. Comprehensive Integration of Single-Cell Data., 177, 1888-190221 (2019).

35. Pedregosa, F. et al. Scikit-learn: Machine Learning in Python. J Mach Learn Res, 12, 2825-2830 (2011).

36. Benjamini, Y. \& Hochberg, Y. Controlling the false discovery rate: a practical and powerful approach to multiple testing. J Roy Stat Soc, B, 57, 289-300 (1995).

37. Wolock, S. L., Lopez, R. \& Klein, A. M. Scrublet: Computational Identification of Cell Doublets in SingleCell Transcriptomic Data. Cell Syst, 8, 281-291 (2019).

38. Jin, S. et al. Inference and analysis of cell-cell communication using CellChat. Nat Commun, 12, 1088 (2021).

39. Chan, M. et al. Identification of substituted pyrimido[5,4-b]indoles as selective toll-like receptor 4 ligands (J Med Chem, 2013). 
40. Nakajima, R. et al. 2018.Protein Microarray Analysis of the Specificity and Cross-Reactivity of Influenza Virus Hemagglutinin-Specific Antibodies. mSphere

41. Van Kerkhove, M. D. Brief literature review for the WHO global influenza research agenda t-highly pathogenic avian influenza H5N1 risk in humans HPAI/H5N1 in humans. Influ Other Respir Viruses, 7, 26-33 (2013).

42. Diehl, S. \& Rincón, M. The two faces of IL-6 on Th1/Th2 differentiation. Mol Immunol, 39, 531-536 (2002).

43. Lu, Y. et al. The interaction of influenza H5N1 viral hemagglutinin with sialic acid receptors leads to the activation of human y $\delta$ T cells. Cell Mol Immunol, 10, 463-470 (2013).

44. Liu, W-C., Lin, S-C., Yu, Y-L., Chu, C-L. \& Wu, S-C. Dendritic Cell Activation by Recombinant Hemagglutinin Proteins of H1N1 and H5N1 Influenza A Viruses. J Virol, 84, 12011-12017 (2010).

45. Venkataraman, C., Schaefer, G. \& Schindler, U. Cutting edge: Chandra, a novel four-transmembrane domain protein differentially expressed in helper type 1 lymphocytes. J Immunol, 165, 632-636 (2000).

46. Zhu, Y., Wang, W. \& Wang, X. 2015. Roles of transcriptional factor 7 in production of inflammatory factors for lung diseases. J Transl Med 2015131 13:1-13

47. Smith, X., Taylor, A. \& Rudd, C. E. T-cell immune adaptor SKAP1 regulates the induction of collageninduced arthritis in mice. Immunol Lett, 176, 122 (2016).

48. Ng, S. S. et al. The NK cell granule protein NKG7 regulates cytotoxic granule exocytosis and inflammation. Nat Immunol, 21, 1205-1218 (2020).

49. Ondr, J. K. \& Pham, C. T. N. Characterization of murine cathepsin W and its role in cell-mediated cytotoxicity. J Biol Chem, 279, 27525-27533 (2004).

50. Kalekar, L. A. \& Mueller, D. L. Relationship between CD4 Tregs and anergy in vivo. J Immunol, 198, 2527 (2017).

51. Yamaguchi, T. et al. Control of Immune Responses by Antigen-Specific Regulatory T Cells Expressing the Folate Receptor., 27, 145-159 (2007).

52. Nechanitzky, R. et al. 2013. Transcription factor EBF1 is essential for the maintenance of B cell identity and prevention of alternative fates in committed cells. Nat Immunol 2013148 14:867-875

53. Su, H., Na, N., Zhang, X. \& Zhao, Y. The biological function and significance of CD74 in immune diseases. Inflamm Res, 66, 209-216 (2017).

54. Bailey, C. C., Zhong, G., Huang, I-C. \& Farzan, M. IFITM-Family Proteins: The Cell's First Line of Antiviral Defense. Annu Rev Virol, 1, 261-283 (2014).

55. Vogl, T. et al. Mrp8 and Mrp14 are endogenous activators of Toll-like receptor 4, promoting lethal, endotoxin-induced shock. Nat Med, 13, 1042-1049 (2007).

56. Lee, B. C. et al. 2017. Selenoprotein MsrB1 promotes anti-inflammatory cytokine gene expression in macrophages and controls immune response in vivo.Sci Rep7 
57. Hovden, A. O., Cox, R. J. \& Haaheim, L. R. Whole influenza virus vaccine is more immunogenic than split influenza virus vaccine and induces primarily an IgG2a response in BALB/c mice. Scand $J$ Immunol, 62, 36-44 (2005).

58. Jeisy-Scott, V. et al. TLR7 recognition is dispensable for influenza virus A infection but important for the induction of hemagglutinin-specific antibodies in response to the 2009 pandemic split vaccine in mice. J Virol2012/07/28, 86, 10988-10998 (2012).

59. Davis, C. W. et al. Influenza vaccine-induced human bone marrow plasma cells decline within a year after vaccination., 370, 237-241 (2020).

60. O'Hagan, D. T. MF59 is a safe and potent vaccine adjuvant that enhances protection against influenza virus infection. Expert Rev Vaccines, 6, 699-710 (2007).

61. Del Giudice, G., Rappuoli, R. \& Didierlaurent, A. M. Correlates of adjuvanticity: A review on adjuvants in licensed vaccines. Semin Immunol, 39, 14-21 (2018).

62. Ansaldi, F. et al. Cross-protection by MF59 ${ }^{\mathrm{TM}}$-adjuvanted influenza vaccine: Neutralizing and haemagglutination-inhibiting antibody activity against $\mathrm{A}(\mathrm{H} 3 \mathrm{~N} 2)$ drifted influenza viruses. Vaccine, 26, 1525-1529 (2008).

63. Banzhoff, A. et al. MF59® -adjuvanted vaccines for seasonal and pandemic influenza prophylaxis. Influenza Other Respi Viruses, 2, 243-249 (2008).

64. Tregoning, J. S., Russell, R. F. \& Kinnear, E. Adjuvanted influenza vaccines. Hum Vaccines Immunother, 14, 550-564 (2018).

65. Galli, G. et al. Fast rise of broadly cross-reactive antibodies after boosting long-lived human memory B cells primed by an MF59 adjuvanted prepandemic vaccine. Proc Natl Acad Sci U S A, 106, 7962-7967 (2009).

66. Young, B. E., Sadarangani, S. P. \& Leo, Y. S. The avian influenza vaccine Emerflu. Why did it fail? Expert Rev Vaccines, 14, 1125-1134 (2015).

67. Garçon, N., Vaughn, D. W., Arnaud \& Didierlaurent M Development and evaluation of AS03, an Adjuvant System containing a-tocopherol and squalene in an oil-in-water emulsion. Expert Rev Vaccines, 11, 349-366 (2014).

68. Didierlaurent, A. M. et al. AS04, an Aluminum Salt- and TLR4 Agonist-Based Adjuvant System, Induces a Transient Localized Innate Immune Response Leading to Enhanced Adaptive Immunity. J Immunol, 183, 6186-6197 (2009).

69. Angelo, M. G. et al. Strategies for continuous evaluation of the benefit-risk profile of HPV-16/18-AS04adjuvanted vaccine. Expert Rev Vaccines, 13, 1297-1306 (2014).

70. Kundi, M. New hepatitis B vaccine formulated with an improved adjuvant system. Expert Rev Vaccines, 6, 133-140 (2007).

71. Lecrenier, N. et al. Development of adjuvanted recombinant zoster vaccine and its implications for shingles prevention. Expert Rev Vaccines, 7, 619-634 (2018).

72. Hemmi, H. et al. A Toll-like receptor recognizes bacterial DNA., 408, 740-745 (2000). 
73. Bode, C., Zhao, G., Steinhagen, F., Kinjo, T. \& Klinman, D. M. Cpg DNA as a vaccine adjuvant. Expert Rev Vaccines, 10, 499-511 (2011).

74. Cooper, C. \& MacKie, D. Hepatitis B surface antigen-1018 ISS adjuvant-containing vaccine: A review of HEPLISAVTM safety and efficacy. Expert Rev Vaccines, 10, 417-427 (2011).

75. Eng, N. F., Bhardwaj, N., Mulligan, R. \& Diaz-Mitoma, F. The potential of 1018 ISS adjuvant in hepatitis B vaccines: HEPLISAV" review. Hum Vaccines Immunother, 9, 1661-1672 (2013).

76. Nagashima, K. \& Mousa, J. 2021. Next-Generation Influenza HA Immunogens and Adjuvants in Pursuit of a Broadly Protective Vaccine. Viruses 13

77. Li, Z., Zhao, Y., Li, Y. \& Chen, X. Adjuvantation of Influenza Vaccines to Induce Cross-Protective Immunity. Vaccines, 9, 1-22 (2021).

78. Wei, C. et al. Next-generation influenza vaccines: opportunities and challenges. Nat Rev Drug Discov, 19, 239-252 (2020).

79. Mutwiri, G. et al. Combination adjuvants: The next generation of adjuvants? Expert Rev Vaccines, 10, 95-107 (2011).

80. Apostolico Jde, S., Lunardelli, V. A., Coirada, F. C., Boscardin, S. B. \& Rosa, D. S. 2016. Adjuvants: classification, modus operandi, and licensing. J Immunol Res 2016:1459394

81. Bidet, K. et al. Mimicking immune signatures of flavivirus infection with targeted adjuvants improves dengue subunit vaccine immunogenicity. npj Vaccines, 4, 27 (2019).

82. Ko, E. J., Lee, Y. T., Lee, Y., Kim, K. H. \& Kang, S. M. Distinct effects of monophosphoryl lipid A, oligodeoxynucleotide $\mathrm{CpG}$, and combination adjuvants on modulating innate and adaptive immune responses to influenza vaccination. Immune Netw, 17, 326-342 (2017).

83. Ko, E. J. et al. MPL and CpG combination adjuvants promote homologous and heterosubtypic cross protection of inactivated split influenza virus vaccine. Antiviral Res, 156, 107-115 (2018).

84. Cao, X. Self-regulation and cross-regulation of pattern-recognition receptor signalling in health and disease. Nat Rev Immunol, 16, 35-50 (2016).

85. Temizoz, B. et al. TLR9 and STING agonists synergistically induce innate and adaptive type-II IFN. Eur $J$ Immunol, 45, 1159-1169 (2015).

86. Kayraklioglu, N., Horuluoglu, B. \& Klinman, D. CpG Oligonucleotides as Vaccine Adjuvants. Methods Mol Biol, 2197, 51-85 (2021).

87. Romerio, A. \& Peri, F. 2020. Increasing the Chemical Variety of Small-Molecule-Based TLR4 Modulators: An Overview. Front Immunol 11.

88. EA, A. B., HS, H., HS, W. J. T., C, S. \& V, J H MyD88-dependent and MyD88-independent pathways in synergy, priming, and tolerance between TLR agonists. J Immunol, 178, 1164-1171 (2007).

89. Garcia-Cordero, J. L., Nembrini, C., Stano, A., Hubbell, J. A. \& Maerkl, S. J. A high-throughput nanoimmunoassay chip applied to large-scale vaccine adjuvant screening. Integr Biol, 5, 650-658 (2013). 
90. Mathew, N. R. \& Angeletti, D. 2020. Recombinant Influenza Vaccines: Saviors to Overcome Immunodominance.Front Immunol10

\section{Tables}

Table 1. Formulations used for adjuvant screen. Groups of female $\mathrm{C} 57 \mathrm{BI} / 6$ mice ( $\mathrm{N}=4$ per group) were administered antigen and TLR agonist(s) to a final volume of $50 \mathrm{~mL}$ in PBS per mouse (groups 1-9) or $25 \mathrm{~mL}$ in PBS and an equal volume of AddaVAX emulsion added (groups 10-18), and administered via the s.c. route (base of tail). 
Antigen TLR agonist

Emulsion

\begin{tabular}{|c|c|c|c|c|c|c|c|c|}
\hline Group & $\begin{array}{l}\mathrm{H} 5 \\
\text { trimer }\end{array}$ & TLR2/6_4_7 & TLR2/6 & TLR4 & TLR7 & $\begin{array}{l}\text { CpG } \\
\text { (TLR9) }\end{array}$ & MPLA(TLR4) & AddaVAX \\
\hline 1 & $2.5 \mathrm{mg}$ & 1 nmole & & & & & & \\
\hline 2 & $2.5 \mathrm{mg}$ & & $\begin{array}{l}1 \\
\text { nmole }\end{array}$ & $\begin{array}{l}1 \\
\text { nmole }\end{array}$ & $\begin{array}{l}1 \\
\text { nmole }\end{array}$ & & & \\
\hline 3 & $2.5 \mathrm{mg}$ & & $\begin{array}{l}1 \\
\text { nmole }\end{array}$ & & & & & \\
\hline 4 & $2.5 \mathrm{mg}$ & & & $\begin{array}{l}1 \\
\text { nmole }\end{array}$ & & & & \\
\hline 5 & $2.5 \mathrm{mg}$ & & & & $\begin{array}{l}1 \\
\text { nmole }\end{array}$ & & & \\
\hline 6 & $2.5 \mathrm{mg}$ & & & & & $\begin{array}{l}1 \\
\text { nmole }\end{array}$ & & \\
\hline 7 & $2.5 \mathrm{mg}$ & & & & & & 3 nmole & \\
\hline 8 & $2.5 \mathrm{mg}$ & & & & & $\begin{array}{l}1 \\
\text { nmole }\end{array}$ & 3 nmole & \\
\hline 9 & $2.5 \mathrm{mg}$ & & & & & & & \\
\hline 10 & $2.5 \mathrm{mg}$ & 1 nmole & & & & & & $25 \mathrm{~mL}$ \\
\hline 11 & $2.5 \mathrm{mg}$ & & $\begin{array}{l}1 \\
\text { nmole }\end{array}$ & $\begin{array}{l}1 \\
\text { nmole }\end{array}$ & $\begin{array}{l}1 \\
\text { nmole }\end{array}$ & & & $25 \mathrm{~mL}$ \\
\hline 12 & $2.5 \mathrm{mg}$ & & $\begin{array}{l}1 \\
\text { nmole }\end{array}$ & & & & & $25 \mathrm{~mL}$ \\
\hline 13 & $2.5 \mathrm{mg}$ & & & $\begin{array}{l}1 \\
\text { nmole }\end{array}$ & & & & $25 \mathrm{~mL}$ \\
\hline 14 & $2.5 \mathrm{mg}$ & & & & $\begin{array}{l}1 \\
\text { nmole }\end{array}$ & & & $25 \mathrm{~mL}$ \\
\hline 15 & $2.5 \mathrm{mg}$ & & & & & $\begin{array}{l}1 \\
\text { nmole }\end{array}$ & & $25 \mathrm{~mL}$ \\
\hline 16 & $2.5 \mathrm{mg}$ & & & & & & 3 nmole & $25 \mathrm{~mL}$ \\
\hline 17 & $2.5 \mathrm{mg}$ & & & & & $\begin{array}{l}1 \\
\text { nmole }\end{array}$ & 3 nmole & $25 \mathrm{~mL}$ \\
\hline 18 & $2.5 \mathrm{mg}$ & & & & & & & $25 \mathrm{~mL}$ \\
\hline
\end{tabular}

Table 2. Summary of the effect of different adjuvants on homo- (H5) and hetero- $\mathrm{H} 1$ ) subtypic crossreactivity and polarization of the Th1 vs Th2 response. Data for homo- and heterosubtypic cross-reactivity from Fig. 1, data for Th1 and Th2 response from Fig. 3. Key: For IgG, IgG1 and IgG2c, median signal 
intensities -, <2,000; +, 2,000-9,999; ++, 10,000-19,999; +++, 20,000-29,999; ++++, 30,000-39,999; +++++, $>40,000$. For numbers of IL-4 and IFN-g spot forming cells (SFCs) per $5 \times 10^{5}$ splenocytes:,$-<10 ;+, 10-24$; ,$++ 25-49 ;+++, 50-75 ;++++,>75$

\begin{tabular}{|c|c|c|c|c|c|c|c|c|}
\hline & \multicolumn{4}{|c|}{ Without AddaVAX } & \multicolumn{4}{|c|}{ With AddaVAX } \\
\hline & & $\operatorname{lgG} \mathrm{H} 1$ & $\lg G 1$ & $\lg G 2 c$ & IgG H5 & IgG H1 & $\lg G 1$ & $\lg G 2 c$ \\
\hline & IgG H5 & & IL-4 & IFN-g & & & IL-4 & IFN-g \\
\hline \multicolumn{9}{|l|}{ Agonist } \\
\hline \multirow[t]{2}{*}{ TLR 2/6_4_7 } & ++ & - & ++ & - & ++ & + & ++++ & - \\
\hline & & & ++ & - & & & + & - \\
\hline \multirow[t]{2}{*}{ TLR 2/6, 4, 7} & - & - & - & - & +++ & ++ & +++++ & + \\
\hline & & & +++ & - & & & ++ & + \\
\hline \multirow[t]{2}{*}{ TLR 2/6 } & + & - & + & - & ++ & + & ++++ & - \\
\hline & & & +++ & - & & & + & - \\
\hline \multirow[t]{2}{*}{ TLR 4} & - & - & - & - & ++++ & ++ & +++++ & + \\
\hline & & & + & - & & & ++++ & - \\
\hline \multirow[t]{2}{*}{ TLR 7} & - & - & ++ & - & +++ & ++ & +++ & + \\
\hline & & & - & + & & & + & - \\
\hline \multirow[t]{2}{*}{ TLR 9} & - & - & - & - & ++++ & ++ & +++ & ++ \\
\hline & & & - & + & & & + & ++++ \\
\hline \multirow[t]{2}{*}{ TLR 4 (MPLA) } & +++ & + & + & ++ & +++ & ++ & +++ & ++ \\
\hline & & & - & +++ & & & ++ & ++++ \\
\hline TLR 9, 4 & +++ & ++ & - & +++++ & ++++ & +++ & + & ++++ \\
\hline (CpG, MPLA) & & & - & ++++ & & & ++++ & ++++ \\
\hline \multirow[t]{2}{*}{ none } & - & - & - & - & +++ & ++ & ++++ & +++ \\
\hline & & & ++ & - & & & ++ & + \\
\hline
\end{tabular}

Figures 

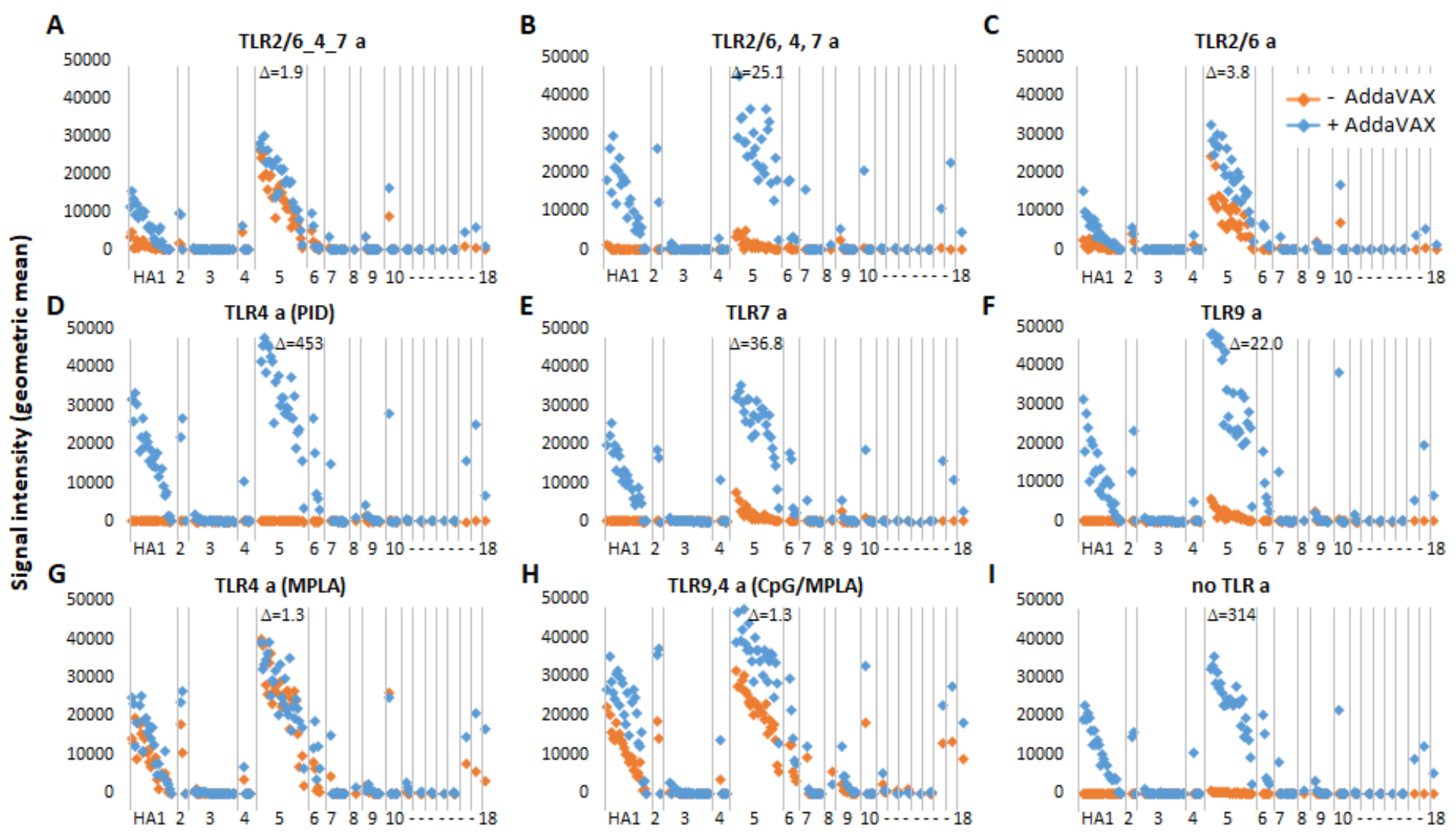

\section{Figure 1}

IgG profiles in response to trimerized influenza virus VN04 H5 vaccine formulated with different TLR agonists, with or without squalene oil-in-water emulsion (AddaVAX). Antibody profiles were determined by probing immune plasma (d28-post vaccination) against HA protein microarray (Supplementary Table S1). Antigens (horizontal axis) are full-length (HAO) HA variants ranked within subtype by overall average of whole study population. Signal intensities (vertical axis) are geometric means of each vaccine group ( $\mathrm{N}=4$ mice). Abbreviations: $\mathrm{CpG}$, cytosine-guanine oligo deoxynucleotide; fold change in $\mathrm{H} 5$ signal intensities when TLRa formulated with AddaVAX; MPLA, monophosphoryl lipid A; PID, pyrimido-indole derivative; TLRa, TLR agonist. 

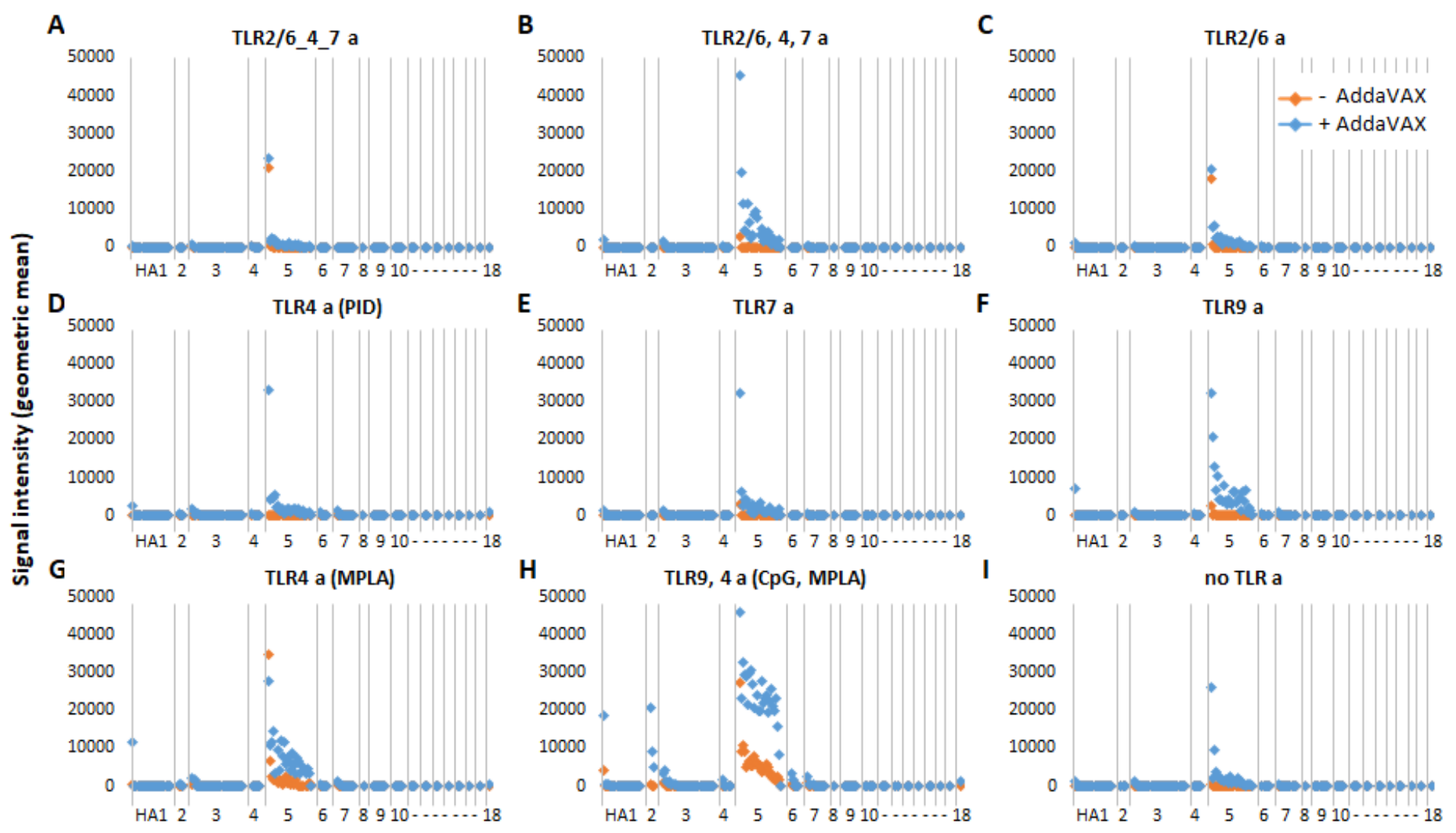

\section{Figure 2}

IgG profiles in response to trimerized influenza virus VN04 H5 vaccine formulated with different TLR agonists, with or without squalene oil-in-water emulsion (AddaVAX). As per Figure 1, except arrayed antigens (horizontal axis) are HA1 regions of HA. Abbreviations, as Fig. 2. 
A

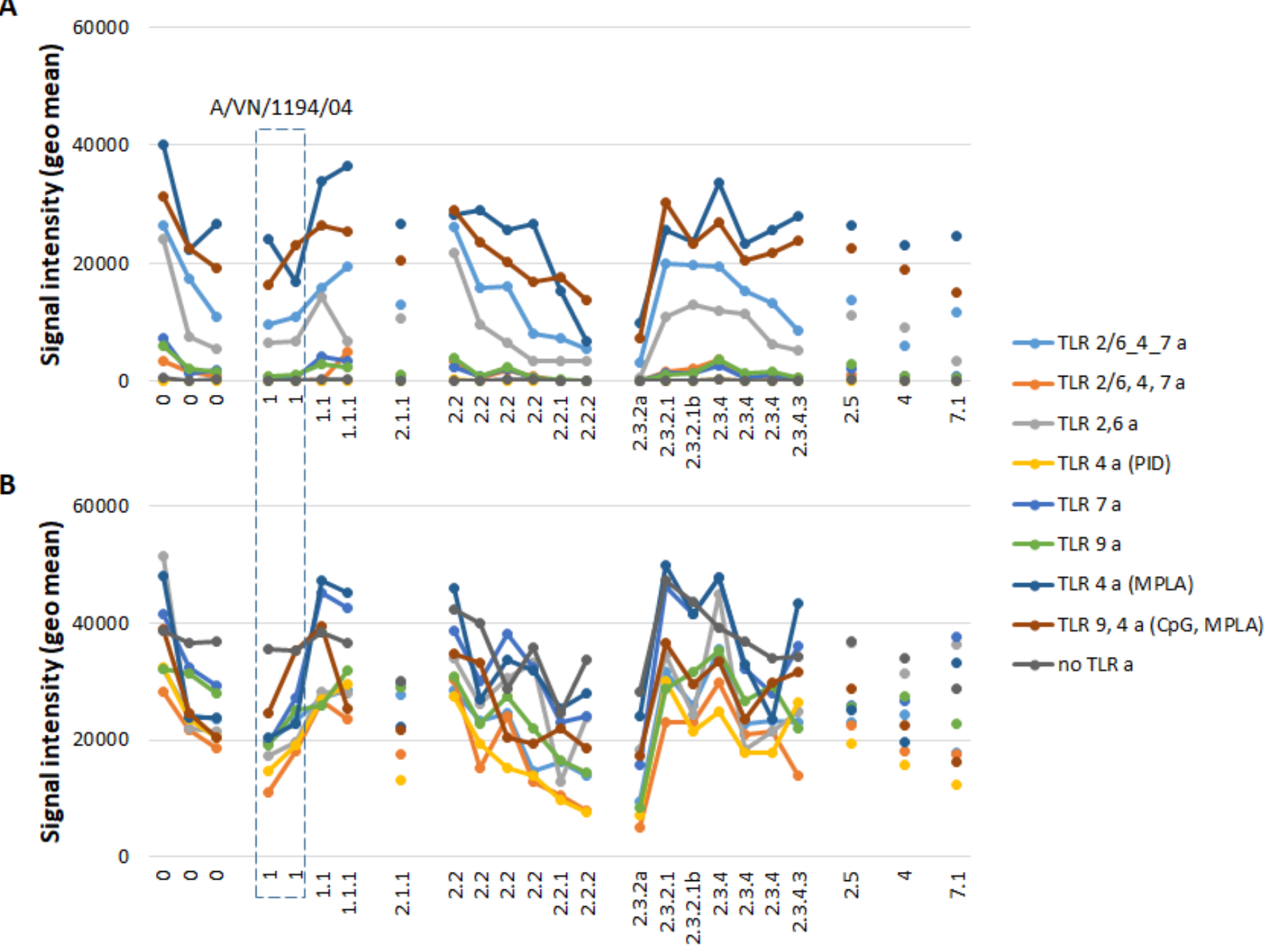

H5 clade

Figure 3

Cross-clade homosubtypic cross-reactivity measured by protein microarray. IgG profiles in response to trimerized influenza virus VN04 (A/VN/1203/2004) H5 vaccine formulated with different TLR agonists, with or without squalene oil-in-water emulsion (AddaVAX). H5 antigens (horizontal axis) are full-length (HA0) HA variants that have been organized by clade (classifications from www.fludb.org). Signal intensities (vertical axis) are geometric means of each vaccine group ( $N=4$ mice). $A$, minus AddaVAX; $B$, with AddaVAX. Boxed, two VN04 H5 proteins on the array (expressed in human or insect cells) that correspond with immunizing H5 isolate. Abbreviations, as Fig. 2. 
A Minus AddaVAX
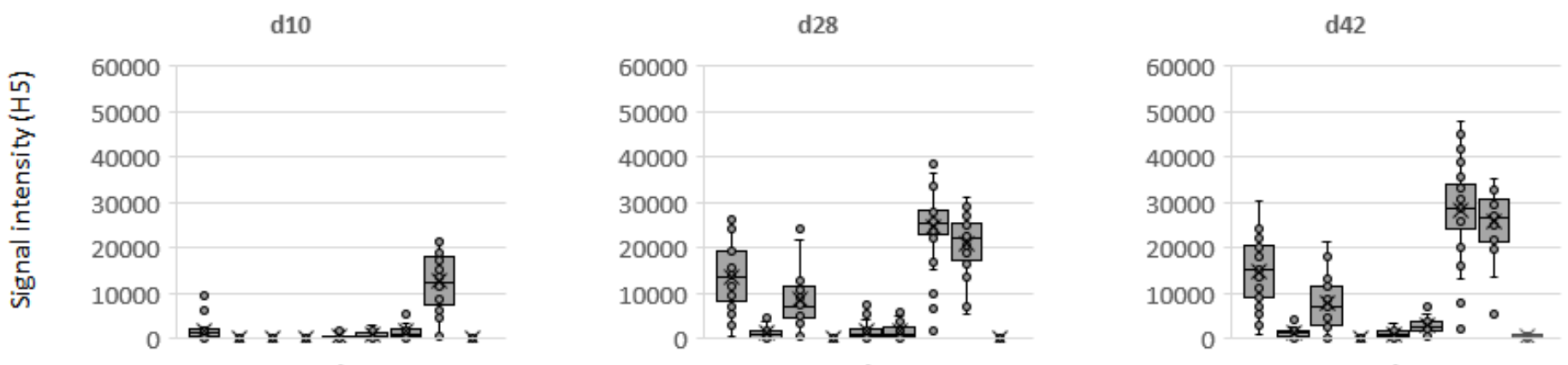

B Plus AddaVAX
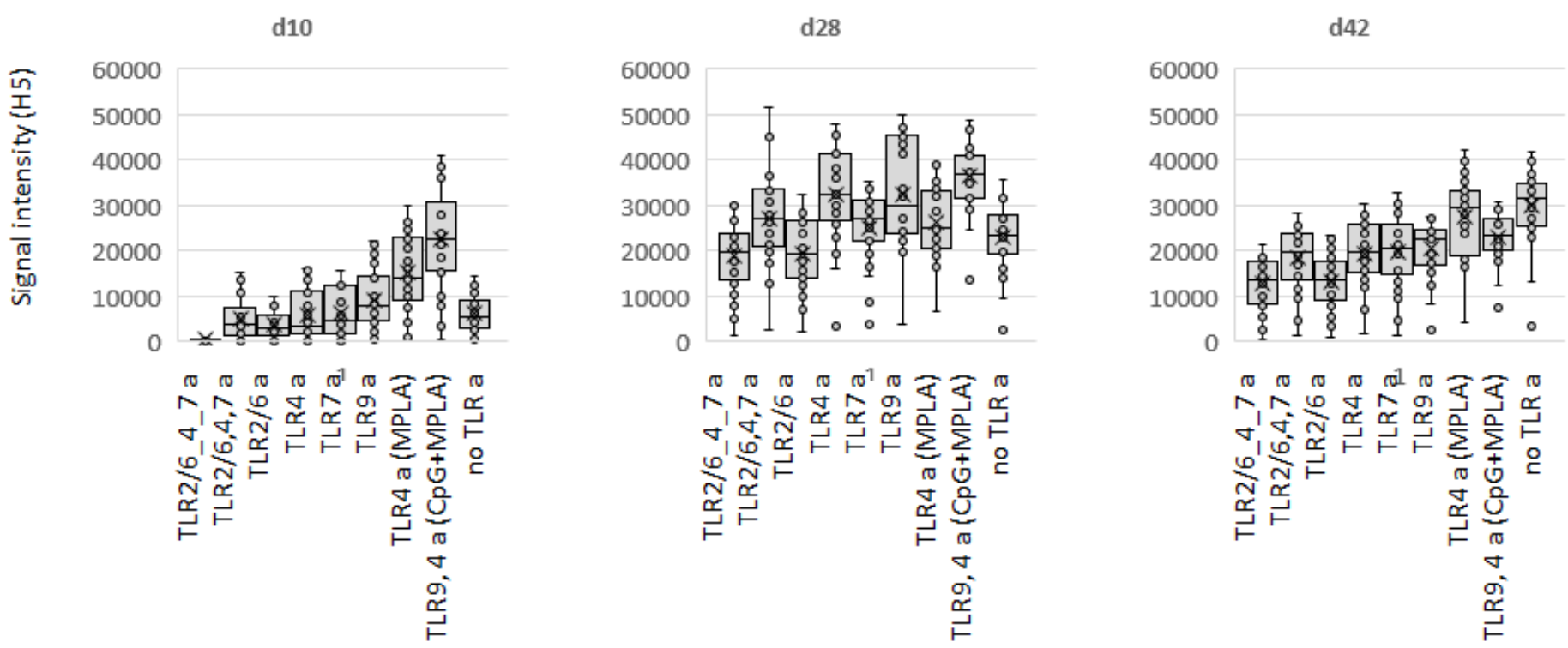

Figure 4

Dynamics of IgG profiles in response to trimerized influenza virus VN04 H5 vaccine formulated with different TLR agonists. Box plots of H5 drift variants; each spot corresponds a different H5 variant on the microarray (geometric mean of 4 mice). Different TLR agonists, listed at the bottom, were administered A) without or B) with squalene oil-in-water emulsion (AddaVAX). Abbreviations, as Fig. 2. 
A

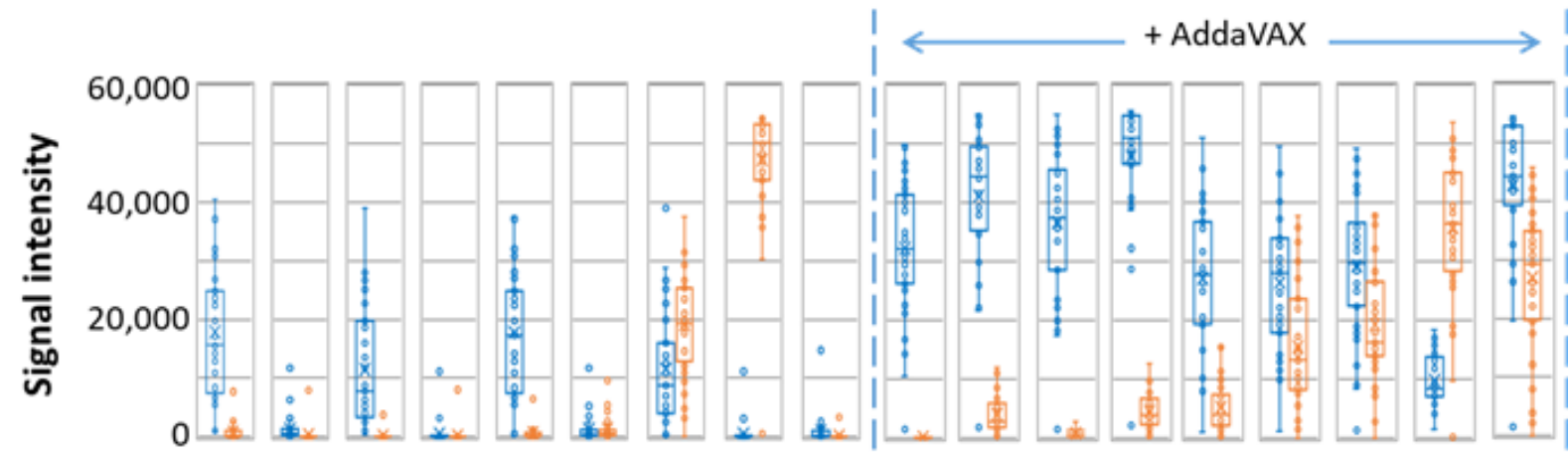

$\square \lg G 1$

$\square \lg 22 c$

B

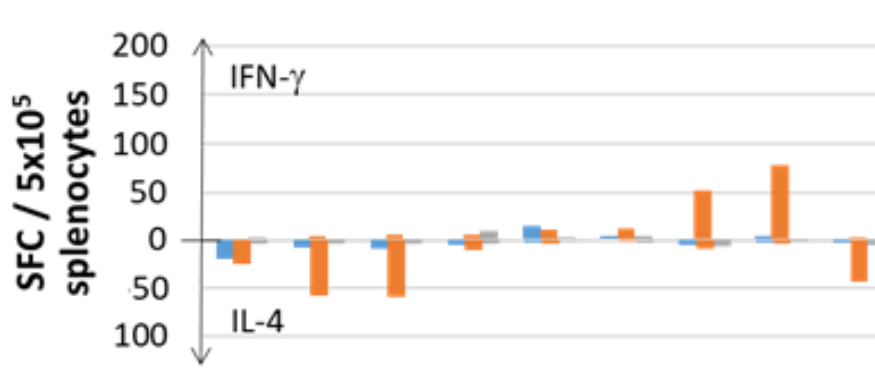

C

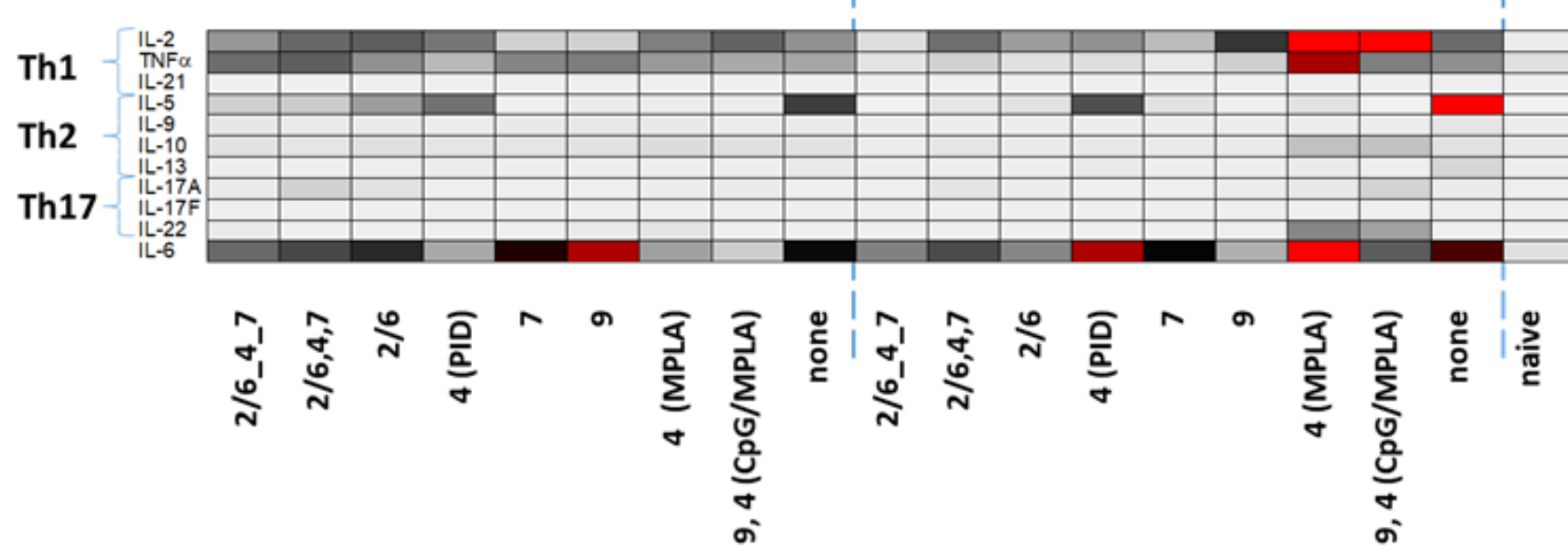

Agonist formulation

\section{Figure 5}

Th1 vs. Th2 balance in response to H5 vaccine formulated with different TLR agonists, with or without AddaVAX. A), Box plots of IgG1 and IgG2c profiles for H5 drift variants; each spot corresponds a different $\mathrm{H} 5$ variant on the microarray (geometric mean of 4 mice). B), spot-forming cells (SFC) in IFN- and IL-4 ELISpots performed on the same mice as shown in A) 10 days post i.p. boost (means of 4 mice), using H1, $\mathrm{H} 5$ and $\mathrm{H} 7$ as recall antigens. Cell viability confirmed using Con-A mitogen (not shown). C) Multiplex cytokine bead array (LEGENDplex ${ }^{\mathrm{TM}}$ ) of culture supernatants of ELISpots shown in B). Other abbreviations, as Fig. 2.

\section{Figure 6}

MPLA and CpG synergize to induce HA-specific plasma cell formation in vivo. Mice were immunized on day 0 with PBS (control), or influenza virus HA (H7 from A/Anhui/1/2013) alone or with MPLA, CpG or 
MPLA/CpG, all in the presence of AddaVAX. Mice were boosted i.p. on day 14, and spleens harvested on day 19. A) plasma cells, defined as CD138+, CD19lo; B) antigen-specific plasma cells; C) numbers of total (blue) and antigen-specific (red) plasma cells; D) H7-specific plasma cells induced by immunization with IVAX1 produce predominantly Th1-associated IgG2a.

\section{Figure 7}

Further characterization of H5/IVAX-1. Mice were administered trimerized H5 antigen from A/Viet Nam/1203/04 in combination adjuvant CpG/MPLA/AddaVAX ("IVAX-1"). (A) Virus microneutralization assay. Five C57BI/ 6 mice were immunized and boosted on days 14 and d42, and plasma samples collected on d10, d28 and d56 tested in microneutralization (MN) assays against reassortant H5N1 virus. Plasma from H5N1-infected mouse ('infected') and plasma from naïve mice (NMS) were used as positive and negative controls, respectively; PB1 and PB2 are post boost 1 and 2, respectively. Percent inhibition of H5N1 virus increases with boosting. (B) IVAX-1 preferentially stimulates CD4+ T cells compared to CD8+ T cells. T cell recall assays were performed by incubating splenocytes from H5/IVAX1-immunized mice with recall antigens as either soluble $\mathrm{H} 5$ protein (monomers from Sinobiological) at different concentrations $(\mathrm{mg} / \mathrm{ml})$, or syngeneic vAPCs (splenocytes exposed for $1 \mathrm{~h}$ to reassortant H5N1 influenza virus) at different splenocyte : VAPC ratios. Cultures were incubated overnight, and the proportions of IFN-positive CD 4 and CD8 cells determined using ICS. (C) H5/IVAX-1 administered by intranasal (i.n.) route induces IgG2C in lung and plasma, and IgA in lung and nasal turbinates. B6 mice were administered H5 trimers in IVAX-1 adjuvant via the i.n. route on d0 and d56. Antibody profiling was performed on d69 using protein microarrays probed with plasma and lung and nasal washes. Antigens are ranked left-to-right in the same order in each panel indicating the IgG and IgA profiles are essentially identical. (D) Administration of H5/IVAX-1 via the i.n. route induces essentially identical profiles to administration via the s.c. route. Shown are scatter plots of Abs signals against different $\mathrm{H} 5$ variants on protein microarrays. Plasma samples were obtained after i.n. and s.c. administration ( $x$ and $y$ axes, respectively); each point represents a different $\mathrm{H} 5$ drift variant. 

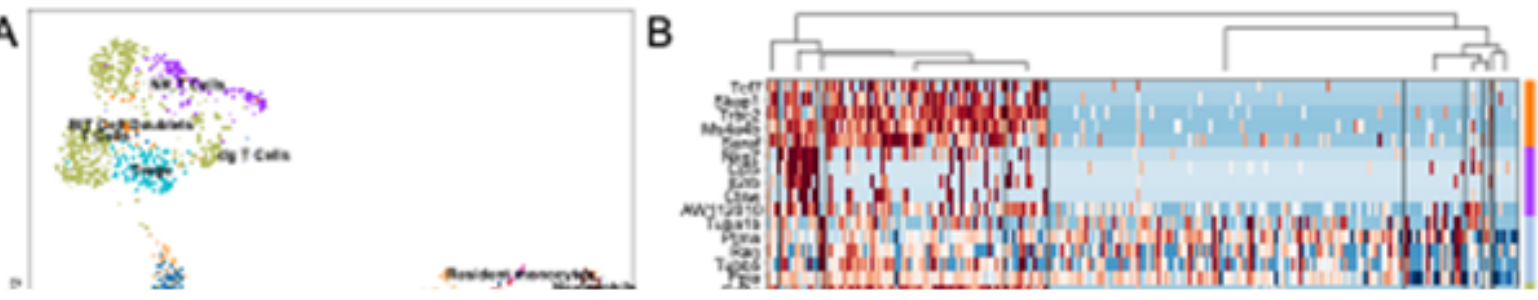

\section{Figure 8}

Analysis of single cell transcriptomic data in unimmunized and H5/IVAX-1 immunized mice. C57BI/6 female mice ( $\mathrm{N}=3$ ) were administered $5 \mathrm{mg} \mathrm{H} 5$ in $50 \mathrm{~mL}$ IVAX-1 adjuvant s.c. and boosted on d14 (s.c.) and d28 (i.p.), and spleens collected 7 days later. Splenocytes from a single age-matched naïve mouse were also prepared for comparison. (A) UMAP visualization of splenocytes before and after vaccination shows transcripts are derived mostly lymphoid lineage with some myeloid cells. (B) Heatmap of differential expression testing; the highest differentially expressed genes for most major populations are their marker genes (see text for details). Each column represents a single cell. (C) Expression of B cell Ighg2c, lghg2b and Ighg1 genes is elevated after immunization, with more IgG2 transcripts compared to IgG1. (D) Mean expression of Ifng gene in CD8+ and CD4+ T cells before and after immunization showing upregulation in 
both subsets. (E) Circular plot showing the main cell-cell interactions in pre- and post-vaccination splenocytes (blue and red lines, respectively).

\section{Supplementary Files}

This is a list of supplementary files associated with this preprint. Click to download.

- SupplementaryFigures.docx 